\title{
Sex differences in cardiovascular, neuroendocrine and behavioral changes evoked by chronic stressors in rats
}

\author{
Jonas O. Vieira ${ }^{\mathrm{a}, \mathrm{b}}$, Josiane O. Duarte ${ }^{\mathrm{a}, \mathrm{b}}$, Willian Costa-Ferreira ${ }^{\mathrm{a}, \mathrm{b}}$, Gessynger Morais-Silva ${ }^{\mathrm{a}, \mathrm{b}}$, \\ Marcelo T. Marin ${ }^{\mathrm{a}, \mathrm{b}}$, Carlos C. Crestani ${ }^{\mathrm{a}, \mathrm{b}, *}$ \\ a Laboratory of Pharmacology, São Paulo State University (UNESP), School of Pharmaceutical Sciences, Araraquara, SP, Brazil \\ b Joint UFSCar-UNESP Graduate Program in Physiological Sciences, São Carlos, SP, Brazil
}

\section{A R T I C L E I N F O}

\section{Keywords:}

Unpredictable stress

Restraint stress

Autonomic activity

Baroreflex

Vascular

HPA axis

\begin{abstract}
A B S T R A C T
This study investigated the physiological, somatic and behavioral changes evoked by daily exposure to the same type of stressor (homotypic) or different aversive stressor stimuli (heterotypic) in male and female rats. For this, adult Wistar rats were subjected to a 10 days regimen of repeated restraint stress (RRS, homotypic stressor) or chronic variable stress (CVS, heterotypic stressor). Effects evoked by CVS included: (i) adrenal hypertrophy and decreased body weight gain in male animals, (ii) a sympathetically-mediated increase in basal heart rate in males, and (iii) a rise in plasma corticosterone concentration and anxiogenic effects in female animals. The homotypic stressor RRS also induced an increase in plasma corticosterone and anxiogenic effects in females, decreased body weight gain in males and evoked a sympathetically-mediated increase in heart rate in both sexes. Changes in cardiovascular function and autonomic activity evoked by both stressors were followed by impairment of baroreflex activity in males, but not female animals. Both chronic stressors evoked changes in blood pressure responsiveness to vasoconstrictor and vasodilator agents in both sexes. Taken together, these results indicate that regardless of chronic stress regimen males are more vulnerable to somatic effects of chronic stressors, while females appear to be more susceptible to neuroendocrine and behavioral changes. Present findings also indicate that females are selectively vulnerable to cardiovascular and autonomic changes evoked by homotypic stressors. Nevertheless, homotypic and heterotypic stressors similarly affect cardiovascular function and autonomic activity in males.
\end{abstract}

\section{Introduction}

The impact of stress is determined by characteristics of the stressor stimulus, such as chronicity, predictability, controllability and severity (Crestani, 2016; Koolhaas et al., 2011; Steptoe and Kivimaki, 2012). An approach widely employed to investigate the influence of predictability on animal models has been to compare the effect of chronic stressors involving daily exposure to the same type of stressor (i.e., homotypic/ predictable) versus different aversive stimuli (i.e., heterotypic/unpredictable) (Crestani, 2016). Typically, these studies have used the chronic variable stress (CVS) as a heterotypic stressor and the repeated restraint stress (RRS) as a homotypic stressor (Crestani, 2016). Studies comparing RRS and CVS have consistently demonstrated that the latter exhibits a more severe impact on somatic parameters (e.g., adrenal hypertrophy and thymic involution), hypothalamic-pituitary-adrenal (HPA) axis activity, and anxiety- and depression-like behaviors (Haile et al., 2001; Magarinos and McEwen, 1995; Marin et al., 2007; Pastor-
Ciurana et al., 2014). Differences on cardiovascular and autonomic changes following exposure to predictable versus unpredictable stressors are less understood, but recent findings indicated a stress typespecific changes (Crestani, 2016). For instance, Duarte et al. (2015) reported mild hypertension in animals exposure to either CVS or RSS. However, increase in cardiac sympathetic activity, resting tachycardia, and baroreflex impairment was only identified following CVS (Duarte et al., 2015). Altogether, these pieces of evidence indicate a more severe impact of CVS than RRS in neuroendocrine, behavioral, somatic, and cardiovascular responses to stress.

Sexual dimorphism is another factor affecting the impact of stress. For instance, incidence of stress-related diseases, such as depression and anxiety, is more prevalent in females (Bekker and van Mens-Verhulst, 2007; Kessler et al., 1994; Weissman et al., 1996), which is possibly related to increased stress sensitivity (Solomon and Herman, 2009). Accordingly, preclinical studies demonstrated that adrenocorticotropin (ACTH) and corticosterone responses during exposure to a variety of

\footnotetext{
* Corresponding author at: Laboratory of Pharmacology, Department of Natural Active Principles and Toxicology, School of Pharmaceutical Sciences, UNESP-Univ Estadual Paulista, Rodovia Araraquara-Jau Km 01 s/n, 14800-903 Araraquara, SP, Brazil.

E-mail address: crestani@fcfar.unesp.br (C.C. Crestani).
} 
stressors is greater in female than male animals (Doremus-Fitzwater et al., 2009; Galea et al., 1997; Handa et al., 1994; Iwasaki-Sekino et al., 2009; Mitsushima et al., 2003; Rivier, 1999). Sex differences in neuroplasticity as consequence of chronic stress have also been reported (Bowman et al., 2003; Carvalho-Netto et al., 2011; Ter Horst et al., 2009), which is likely related to differential stress responsivity and susceptibility to diseases in males and females. These results provide evidence of sex differences in impact of stress on neuroendocrine function, neuroplasticity, and behavioral disorders.

Despite the evidence of differences on cardiovascular responses during acute sessions of stress in females versus males (Anishchenko et al., 2007; Azar et al., 2005; Eikelis and Van Den Buuse, 2000; Weinstock et al., 1998), influence of the gender on cardiovascular dysfunctions evoked by chronic stressors is poorly understood. Previous studies documented that chronic crowding stress increased blood pressure in hypertensive, but not in normotensive, male rats without affecting this measurement in females (Bernatova et al., 2010; Ledvenyiova-Farkasova et al., 2015). Additionally, Stanley et al. (2014) reported that impairment of vascular reactivity to cholinergic agonists in conduit arteries and skeletal muscle arterioles evoked by CVS was reduced in female versus male mice. However, sex differences in changes on autonomic activity and baroreflex function following exposure to chronic stressors have never been evaluated. This is a relevant issue since it has been proposed that the protective effect of ovarian hormones on cardiovascular function, which is likely related to the reduced prevalence of cardiovascular diseases in young females versus young males (Burt et al., 1995; Kearney et al., 2005), is mediated by an influence of these hormones on vascular function, baroreflex activity, and autonomic activity (dos Santos et al., 2014; Hay, 2016). Additionally, an influence of predictability of the stressor stimulus on its cardiovascular effects has never been evaluated in females. Therefore, our purpose in the present study was to investigate the physiological and somatic changes, with a focus on cardiovascular function, caused by the exposure to homotypic and heterotypic chronic stress regimens in female and male rats.

\section{Material and methods}

\subsection{Animals}

Forty-three females and forty-two males Wistar rats 60-days-old (adult) were used in the present study. Animals were obtained from the animal breeding facility of the São Paulo State University-UNESP (Botucatu, SP, Brazil) and were housed in collective plastic cages (3-4 rats/cage) in a temperature-controlled room at $24^{\circ} \mathrm{C}$ in the Animal Facility of the Laboratory of Pharmacology-UNESP. They were kept under a 12:12 h light-dark cycle (lights on between 7:00 a.m. and 7:00 p.m.) with free access to water and standard laboratory food. Housing conditions and experimental procedures were carried out following protocols approved by local Ethical Committee for Use of Animal, which complies with Brazilian and international guidelines for animal use and welfare.

\subsection{Chronic stress regimens}

The chronic stress protocols were chosen based on previous studies from our group (Costa-Ferreira et al., 2016; Duarte et al., 2015; Marin et al., 2007). Therefore, RRS was chosen as a homotypic stressor, whereas CVS was used as a heterotypic stressor. The animals of RRS group were restrained in opaque plastic cylinders $(15 \mathrm{~cm}$ length and $5.5 \mathrm{~cm}$ internal diameter) for $1 \mathrm{~h}$ daily starting at 10:00 a.m. for 10 consecutive days. The CVS protocol consisted of exposure to different stressors in a variable schedule for 10 consecutive days (Duarte et al., 2015; Marin et al., 2007). The stressors used in the CVS included: 1) restraint stress (60 min); 2) humid sawdust (overnight or all day); 3) cold $\left(4{ }^{\circ} \mathrm{C}\right)$ or room temperature isolation housing; 4) food/water deprivation (overnight); 5) swim stress (4 min); 6) lights on overnight; and 7) lights off during day (120-180 min) (Costa-Ferreira et al., 2016; Duarte et al., 2015; Marin et al., 2007). All the stress sessions were performed in an adjacent room to the animal facility. The RRS and CVS started simultaneously, and during this period animals of control groups were left undisturbed, except for cleaning the cages and body weight measurements, in the animal facility.

\subsection{Experimental design}

2.3.1. Experiment 1: effects of CVS and RRS in female and male rats on anxiety-like behaviors

Female and male animals were randomly divided into three groups: (i) control, (ii) RRS, and (iii) CVS ( $n=8$ /group). The animals of both RRS and CVS groups were submitted to daily sessions of stress for 10 consecutive days. Since the purpose of the present study was to investigate the enduring effects evoked by chronic stress exposure, behavioral tests were performed on the day following the last stress session. Indeed, $24 \mathrm{~h}$ after the last stress session animals in all experimental groups were tested in the elevated plus maze (EPM), and $4 \mathrm{~h}$ later behaviors in the open field (OF) were evaluated. A schematic representation of the complete protocol for investigation of the impact of chronic stressors on anxiety-like behaviors in females and males is presented in Fig. 1.

\subsubsection{Experiment 2: effects of CVS and RRS in female and male rats on} somatic, neuroendocrine, autonomic and cardiovascular parameters

Female and male animals were randomly divided into three groups: (i) control, (ii) RRS, and (iii) CVS ( $n=6-7 /$ group). The animals of RRS and CVS groups were submitted to daily sessions of stress for 10 consecutive days. At 10th day, after the last session of stress, animals in all

\section{Experiment 1: Effects of CVS and RRS in female and male rats on anxiety-like behaviors}
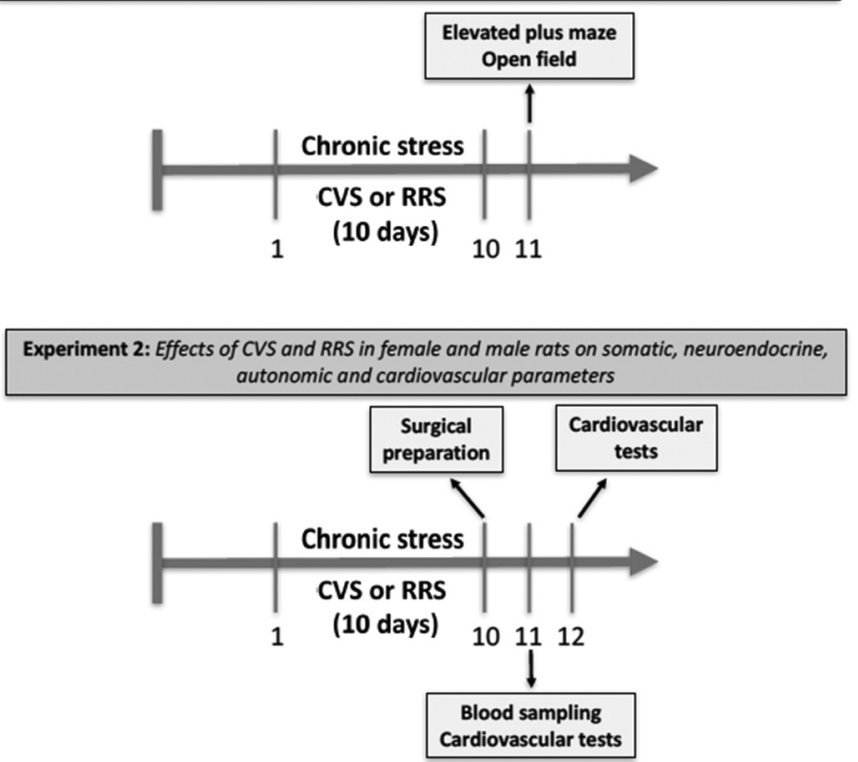

Fig. 1. Schematic representation of the protocols for the investigation of the effects of RRS and CVS in male and female rats. (Top) To investigation of the effects of chronic stressors on anxiety-like behaviors (Experiment 1), animals of RRS and CVS groups were submitted to daily sessions of stress for 10 days. Twenty-four hours after the last session of stress animals in all experimental groups were tested in the EPM and OF. (Bottom) To evaluation of the effects of chronic stressors on neuroendocrine, somatic and cardiovascular/autonomic parameters (Experiment 2), animals of RRS and CVS groups were submitted to daily sessions of stress for 10 days. At 10th day, after the last session of stress, animals in all experimental groups were subjected to surgical preparation. Blood sampling and cardiovascular tests were performed $24 \mathrm{~h}$ after the last stress session in conscious animals. Cardiovascular tests were also performed $48 \mathrm{~h}$ after ending the stress protocols. For details, see description of experimental protocols in the text. 
experimental groups were subjected to surgical preparation. Since the purpose of the present study was to investigate the enduring cardiovascular and autonomic changes evoked by chronic stress exposure, the tests were performed $24 \mathrm{~h}$ and $48 \mathrm{~h}$ after the last stress session in conscious animals.

On first test day, animals were transferred to the experimental room in their home box, and a blood sample $(200 \mu \mathrm{l})$ was collected from the femoral artery cannula between 8 a.m. and 9 a.m. randomly between the experimental groups. Afterwards, animals were allowed $60 \mathrm{~min}$ to adapt to experimental room conditions, such as sound and illumination, before the onset of cardiovascular recording. Then, animals were individually connected to the cardiovascular recording system and subjected to a 30-minute period of basal cardiovascular recording. In the sequence, randomized intravenous infusions of phenylephrine, SNP, and acetylcholine were performed for evaluation of blood pressure changes evoked by each vasoactive agent and analysis of baroreflex function. After that, animals received intravenous administration of methylatropine and propranolol in a random order for determination of cardiac autonomic activity. On the second day, animals were treated with methylatropine and propranolol in the opposite sequence to that realized on the first day. At the end of the experiments, animals were euthanized by anesthetic overdose (urethane, $250 \mathrm{mg} / \mathrm{ml} / 200 \mathrm{~g}$ body weight, i.p.) and their heart, adrenals and thymus were removed and weighed. A schematic representation of the complete protocol for investigation of the impact of chronic stressors on somatic, neuroendocrine and cardiovascular/autonomic parameters in females and males is presented in Fig. 1.

\subsection{Elevated plus maze}

The elevated plus maze (EPM) test was conducted as described before (Gouveia et al., 2016; Padovan and Guimaraes, 2000). Briefly, the apparatus consisted of two opposite open arms $[(50 \mathrm{~cm}$ (length) $\times 10 \mathrm{~cm}$ (width) $\times 0.25 \mathrm{~cm}$ (height)] crossed at a right angle by two arms of the same dimensions enclosed by $40 \mathrm{~cm}$ high walls with no roof. The arms were connected by a common central platform [10 cm (length) $\times 10 \mathrm{~cm}$ (width)], and the maze was located $50 \mathrm{~cm}$ above the floor. Rats were individually placed in the center of the apparatus and were allowed to explore freely the EPM for $5 \mathrm{~min}$.

Rodents naturally avoid the open arms of the EPM, and anxiolytic compounds typically increase the exploration of these arms without changing the number of enclosed-arm entries (Pellow and File, 1986). Therefore, behavioral measures were the frequency of closed-arm entries (CE) and the percentage of open-arm entries (\%OE) and percentage of open-arm time (\%OT). All sessions were videotaped and analysis was realized in a blinded manner using the software Any-maze ${ }^{\circledast}$ (Stoelting, Wood Dale, Illinois, USA).

\subsection{Open field}

The open field (OF) test was used for evaluation of exploratory and anxiety-like behaviors (Grippo et al., 2014; Prut and Belzung, 2003). The OF consisted of a Plexiglas chamber measuring $54 \mathrm{~cm}$ (width) $\times 54 \mathrm{~cm}$ (length) $\times 30 \mathrm{~cm}$ (height). A central area in the middle of the arena measuring $24 \mathrm{~cm}$ (width) $\times 24 \mathrm{~cm}$ (length) was defined as an exposed field and is referred as "center". Rats were individually placed in the middle of the arena and were allowed to explore freely the OF for $5 \mathrm{~min}$.

Such as for EPM, the OF exploit the natural aversion of rodents to exposed fields. Indeed, rodents naturally avoid the central portion of the apparatus, and anxiolytic compounds typically increase the exploration in the central area (Prut and Belzung, 2003). Therefore, analysis of anxiety-like behaviors included measures of the distance travelled (central locomotion) and time spent in the center (center time). The total distance travelled (i.e., center + periphery) (total locomotion) was used as an index of general activity. All sessions were videotaped and analysis was realized in a blinded manner using the software Any-maze ${ }^{\circledast}$ (Stoelting, Wood Dale, Illinois, USA).

\subsection{Surgical preparation}

Animals were anesthetized with tribromoethanol (250 mg/kg, i.p.) and a polyethylene cannula (a $4 \mathrm{~cm}$ segment of PE-10 heat-bound to a $13 \mathrm{~cm}$ segment of PE-50) (Clay Adams, Parsippany, NJ, USA) filled with a solution of heparin (50UI/ml, Hepamax-S ${ }^{\circledR}$, Blausiegel, Cotia, SP, Brazil) diluted in saline $(0.9 \% \mathrm{NaCl})$ was implanted into the abdominal aorta through the femoral artery for cardiovascular recording and blood sampling. A second cannula filled with heparin solution was inserted into the femoral vein for infusion of drugs. Both catheters were tunnelled under the skin and exteriorized on the animal's dorsum. After the surgery, rats received a poly-antibiotic formulation containing streptomycin and penicillin $(560 \mathrm{mg} / \mathrm{ml} / \mathrm{kg}$, i.m.) to prevent infection, and were treated with the non-steroidal anti-inflammatory drug flunixin meglumine $(0.5 \mathrm{mg} / \mathrm{ml} / \mathrm{kg}$, s.c.) for postoperative analgesia.

\subsection{Measurement of cardiovascular parameters}

The cannula implanted into the femoral artery was connected to a pressure transducer (DPT100, Utah Medical Products Inc., Midvale, UT, USA). Pulsatile arterial pressure (PAP) was recorded using an amplifier (Quad Bridge Amp, ML224, ADInstruments, NSW, Australia) and an acquisition board (PowerLab 4/30, ML866/P, ADInstruments, NSW, Australia) connected to a personal computer. Mean (MAP), systolic (SAP), and diastolic (DAP) arterial pressure and HR values were derived from PAP recordings.

\subsection{Assessment of cardiac autonomic activity and intrinsic $H R$}

Cardiac autonomic activity and intrinsic heart rate (iHR) were assessed by intravenously administrating the muscarinic receptor antagonist methylatropine $(3 \mathrm{mg} / \mathrm{ml} / \mathrm{kg})$ and the $\beta$-adrenoceptor antagonist propranolol (4 mg/ml $/ \mathrm{kg}$ ) (Almeida et al., 2015; Duarte et al., 2015). The protocol was performed on two days. On the first day, animals in all experimental groups received intravenous administration of methylatropine and propranolol in a random order. The interval of treatment between drugs was $10 \mathrm{~min}$. Twenty-four hours later, animals were treated with methylatropine and propranolol in the opposite sequence to that performed on the first day.

The parasympathetic activity was determined by analysing the change in basal HR caused by methylatropine while the sympathetic activity was obtained from the HR response evoked by propranolol treatment. The iHR was obtained after combined treatment with propranolol and methylatropine on first and second days, and a mean of the two values was calculated for each animal.

\subsection{Infusion of vasoactive agents}

Intravenous infusion of the $\alpha_{1}$-adrenoceptor agonist phenylephrine $(70 \mu \mathrm{g} / \mathrm{ml}$ at $0.4 \mathrm{ml} / \mathrm{min} / \mathrm{kg})$, the nitric oxide donor sodium nitroprusside (SNP) $(100 \mu \mathrm{g} / \mathrm{ml}$ at $0.8 \mathrm{ml} / \mathrm{min} / \mathrm{kg})$, and acetylcholine $(10 \mu \mathrm{g} / \mathrm{ml}$ at $1.2 \mathrm{ml} / \mathrm{min} / \mathrm{kg})$ was performed using an infusion pump (K.D. Scientific, Holliston, MA, USA) (Cruz et al., 2016; Engi et al., 2016). Phenylephrine evoked incremental pressor response, whereas SNP and acetylcholine caused incremental depressor effects. Infusions of vasoactive drugs were randomized and the second treatment was not realized before cardiovascular parameters returned to control values (interval between infusions was approximately $5 \mathrm{~min}$ ). Infusions lasted for 20-30 s, resulting in the injection of a total dose of $9-14 \mu \mathrm{g} / \mathrm{kg}$ of phenylephrine, $26-40 \mu \mathrm{g} / \mathrm{kg}$ of SNP, and $4-6 \mu \mathrm{g} / \mathrm{kg}$ of acetylcholine. 


\subsection{Assessment of baroreflex activity}

For assessment of baroreflex function, reflex HR changes $(\triangle \mathrm{HR})$ corresponding to MAP changes ( $\triangle \mathrm{MAP}$ ) (each $5 \mathrm{mmHg}$ of $\triangle \mathrm{MAP}$ ) evoked by intravenous infusion of phenylephrine and SNP were plotted to generate sigmoid logistic functions (Crestani et al., 2010; Engi et al., 2016). Analysis of sigmoid curves were characterized by 5 parameters: (i) lower HR plateau $\left(\mathrm{P}_{1}\right.$, in bpm), which is the maximum reflex bradycardia during blood pressure increase; (ii) upper $\mathrm{HR}$ plateau $\left(\mathrm{P}_{2}\right.$, in bpm), which is the maximum reflex tachycardia to blood pressure decrease; (iii) HR range (bpm), which is the difference between $\mathrm{P}_{1}$ and $\mathrm{P}_{2}$; (iv) median blood pressure $\left(\mathrm{BP}_{50}, \mathrm{mmHg}\right)$, which is the MAP at $50 \%$ of the HR range; and (v) average gain ( $\mathrm{G}, \mathrm{bpm} / \mathrm{mmHg}$ ), which is the average slope of the curves between +1 and -1 standard derivations from $\mathrm{BP}_{50}$ (Crestani et al., 2010; Engi et al., 2016). Additionally, from the same points obtained from the correlation of $\triangle \mathrm{MAP}$ and the corresponding reflex HR response $(10,20,30$, and $40 \mathrm{mmHg}$ of $\triangle \mathrm{MAP})$, linear regression curves were created to individual analysis of baroreflex function during the increase and decrease of blood pressure (Crestani et al., 2010).

\subsection{Blood pressure response to vasoactive agents}

The graded MAP changes ( $\triangle \mathrm{MAP}$ ) evoked by intravenous infusion of phenylephrine, SNP, and acetylcholine were plotted to generate dose-effect curves (Cruz et al., 2016; Duarte et al., 2015; Engi et al., 2016). Dose-response curves were generated for each vasoactive agent by calculating the amount of drug infused and the $\triangle \mathrm{MAP}$ each $2 \mathrm{~s}$ after starting the infusion. Analysis of dose-response curves was characterized by 2 parameters: the maximal effect $\left(\mathrm{E}_{\max }\right)$ and the dose at $50 \%$ of the MAP range $\left(E D_{50}\right)$.

\subsection{Plasma corticosterone measurement}

A blood sample $(200 \mu \mathrm{l})$ was collected from the femoral artery cannula for determination of plasma corticosterone concentration. Blood was collected in plastic tubes containing $5 \mu$ l of heparin (5000 UI/ml) (Hepamax-S ${ }^{\circledR}$, Blausiegel, Cotia, SP, Brazil). Samples were centrifuged at $2000 \mathrm{~g}$ for $10 \mathrm{~min}$ at $4{ }^{\circ} \mathrm{C}$ and plasma was stored at $-20^{\circ} \mathrm{C}$ until corticosterone assay was carried out.

Plasma corticosterone concentration was measured by radioimmunoassay, as described previously (Sarnyai et al., 1992). Briefly, $20 \mu \mathrm{l}$ of plasma was diluted 50 times with $0.01 \mathrm{M}$ PBS and placed in a water bath at $75{ }^{\circ} \mathrm{C}$ for $1 \mathrm{~h}$ for heat inactivation of corticosteroid binding globulin. One hundred microliters of a solution of antibody and (3H)-corticosterone (New England Nuclear; Boston, MA, USA) $(10,000-20,000 \mathrm{cpm} / \mathrm{ml})$ was added to each sample, mixed and incubated overnight at $4{ }^{\circ} \mathrm{C}$. Dextran-coated charcoal was used to adsorb free steroid after incubation. Tubes were centrifuged at $2000 \times g$ for $15 \mathrm{~min}$ at $4^{\circ} \mathrm{C}$, the supernatant from each tube was transferred to scintillation vials and the radioactivity was quantified by liquid scintillation spectrometry. Standard curves were constructed using 25,50 , $100,250,500,750,1000$ and $2000 \mathrm{pg} / 100 \mu \mathrm{l}$ of corticosterone (SigmaAldrich, St. Louis, MO, USA).

\subsection{Drugs and solutions}

Propranolol hydrochloride (Sigma-Aldrich, St. Louis, MO, USA), methylatropine (Sigma-Aldrich), phenylephrine hydrochloride (SigmaAldrich), sodium nitroprusside dihydrate (Sigma-Aldrich), acetylcholine (Sigma-Aldrich), tribromoethanol (Sigma-Aldrich), and urethane (Sigma-Aldrich) were dissolved in saline $(0.9 \% \mathrm{NaCl})$. Flunixin meglumine (Banamine ${ }^{\circledast}$, Schering-Plough, Cotia, SP, Brazil) and the polyantibiotic preparation (Pentabiotico ${ }^{\circledR}$, Fort-Dodge, Brazil) were used as provided.

\subsection{Data analysis}

Data were expressed as mean \pm SEM. All data were analyzed by using the software GraphPad Prism version 6.0 (GraphPad Software Inc., La Jolla, CA, USA). Analysis of body weight values was performed using three-way ANOVA, with stress (control, RRS, and CVS) and sex (female vs male) as independent factors and time as repeated measurement. The other measures were analyzed using two-way ANOVA, with stress and sex as independent factors. Planned comparisons using the Bonferroni post-hoc test were performed after two- and three-way ANOVA to assess specific differences between the experimental groups. $P<0.05$ was assumed as significant.

\section{Results}

\subsection{Effects of CVS and RRS in female and male rats on anxiety-like} behaviors

\subsubsection{Elevated plus maze}

Analysis of the number of entries in the enclosed arms indicated a stress $\times$ sex interaction $\left(\mathrm{F}_{(2,42)}=4, P<0.03\right)$, but without main effect of either stress $\left(\mathrm{F}_{(2,42)}=0.5, P>0.05\right)$ or $\operatorname{sex}\left(\mathrm{F}_{(1,42)}=1\right.$, $P>0.05$ ) (Fig. 2). However, post-hoc analysis did not reveal any difference between the experimental groups $(P>0.05)$ (Fig. 2).

Analysis of the percentage of open-arm time indicated effect of stress $\left(\mathrm{F}_{(2,42)}=5, P<0.01\right)$ and a stress $\mathrm{x}$ sex interaction $\left(\mathrm{F}_{(2,42)}=6\right.$, $P<0.007)$, but without main effect of $\operatorname{sex}\left(\mathrm{F}_{(1,42)}=0.001, P>0.05\right)$ (Fig. 2). Analysis of the percentage of open-arm entries indicated a

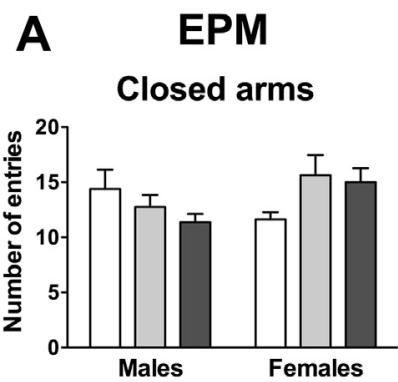

\section{B Open field}
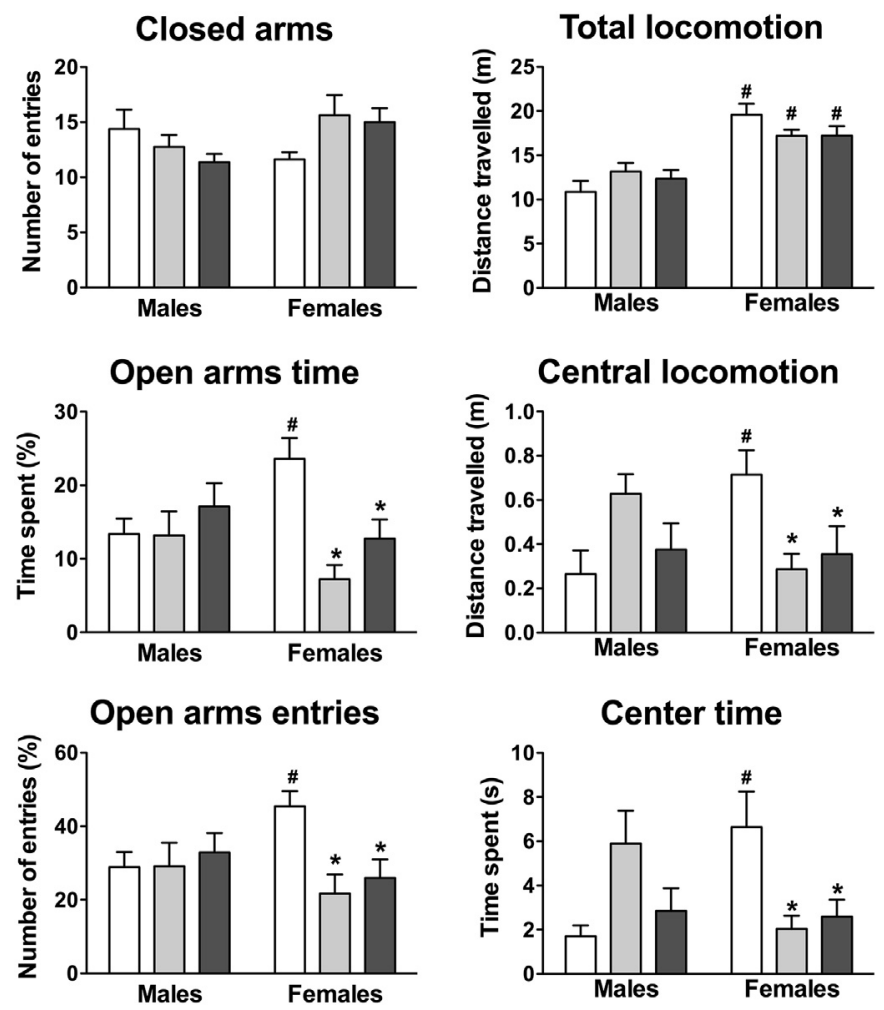

Fig. 2. Behavioral analysis in the elevated plus-maze (EPM) and in the open field test in male and female rats control (white) and subjected to RRS (light grey) or CVS (dark grey). (A) Number of entries in the enclosed arms and percentage of open-arm time and entries in the EPM. (B) Total locomotion (distance travelled in the periphery + center) and distance travelled (central locomotion) and time spent (center time) in the center on open field apparatus. The bars represent the mean \pm SEM. ${ }^{*} P<0.05$ vs respective control group, $\# P<0.05$ vs respective male group. Two-way ANOVA followed by Bonferroni post-hoc test ( $n=8$ /group). 
stress $\times$ sex interaction, but without main effect of either stress $\left(\mathrm{F}_{(2,42)}=3, P>0.05\right)$ or sex $\left(\mathrm{F}_{(1,42)}=0.03, P>0.05\right)$ (Fig. 2). Posthoc analysis revealed that the percentage of time $(P<0.05)$ and number of entries in the open arms $(P<0.05)$ were greater in female of control group, when compared with the respective male group (Fig. 2). Additionally, both RRS and CVS decreased the time spent (RRS: $P<0.05$, CVS: $P<0.05)$ and the number of entries in the open arms (RRS: $P<0.05$, CVS: $P<0.05$ ) in females, but without affecting the open arms exploration in male animals (Fig. 2).

\subsubsection{Open field}

Analysis of the distance travelled and the time spent in the center indicated a stress $\mathrm{x}$ sex interaction (distance: $\mathrm{F}_{(2,42)}=7, P<0.002$; time: $\left.\mathrm{F}_{(2,42)}=8, P<0.0009\right)$, but without main effect of either stress (distance: $\mathrm{F}_{(2,42)}=0.8, P>0.05$; time: $\mathrm{F}_{(2,42)}=1, P>0.05$ ) or sex (distance: $\mathrm{F}_{(1,42)}=0.2, \quad P>0.05 ;$ time: $\mathrm{F}_{(1,42)}=1, \quad P>0.05$ ) (Fig. 2). Post-hoc analysis revealed that both the distance travelled $(P<0.05)$ and the time spent $(P<0.05)$ in the center were greater in female of control group, when compared with the respective male group (Fig. 2). Besides, both RRS $(P<0.05)$ and CVS $(P<0.05)$ decreased the distance travelled and the time in the center in females, but without affecting the central exploration in male animals (Fig. 2).

Analysis of total distance travelled (i.e., center + periphery) (total locomotion) indicated effect of sex $\left(\mathrm{F}_{(1,42)}=47, P<0.0001\right)$, but without influence of stress $\left(\mathrm{F}_{(2,42)}=0.1, P>0.05\right)$ and stress $\times$ sex interaction $\left(\mathrm{F}_{(2,42)}=3, P>0.05\right)$ (Fig. 2). Post-hoc analysis revealed that total locomotion was greater in all groups of females relative to the respective male groups (Fig. 2).

\subsection{Effects of CVS and RRS in female and male rats on somatic parameters} and plasma corticosterone concentration

\subsubsection{Body weight}

Time course analysis of body weight indicated a significant effect of sex $\left(\mathrm{F}_{(1,33)}=38, P<0.0001\right)$ and time $\left(\mathrm{F}_{(2,66)}=72, P<0.0001\right)$, but without effect of stress $\left(\mathrm{F}_{(2,33)}=2, P>0.05\right)$ (Fig. 3A). Additionally, it was identified a sex $\times$ time $\left(\mathrm{F}_{(2,66)}=18, P<0.0001\right)$ and stress $\mathrm{x}$ time $\left(\mathrm{F}_{(4,66)}=5, P<0.004\right)$ interactions, but without a sex $\times$ stress interaction $\left(\mathrm{F}_{(2,33)}=1, P>0.05\right)$. Post-hoc analysis revealed that females in all experimental groups had lower body weight in relation to respective male groups on days $5(P<0.05)$ and 10 $(P<0.05)$ (Fig. 3A). Furthermore, RRS $(P<0.05)$ and CVS $(P<0.05)$ reduced the body weight gain in male animals, which was identified by reduced body weight values at 5 th and 10th days of stress protocols (Fig. 3A). However, neither RRS $(P>0.05)$ nor CVS $(P>0.05)$ affected body weight of females (Fig. 3A).

Analysis of body weight change (i.e., difference between the values at first and 10th day of stress protocol) indicated a significant effect of $\operatorname{sex}\left(\mathrm{F}_{(1,31)}=23, P<0.0001\right)$ and stress $\left(\mathrm{F}_{(2,31)}=5, P<0.009\right)$, but without gender $\mathrm{x}$ stress interaction $\left(\mathrm{F}_{(2,31)}=2, P>0.05\right)$ (Fig. 3B). Post-hoc analysis revealed reduced values in female animals of control $(P<0.05)$ and RRS $(P<0.05)$ groups in relation to respective male groups (Fig. 3B). Moreover, RRS $(P<0.05)$ and CVS $(P<0.01)$ reduced the body weight gain in males (Fig. 3B).

\subsubsection{Heart weight}

Analysis of relative heart weight (heart weight/body weight) indicated a main effect of sex $\left(\mathrm{F}_{(1,31)}=5, P<0.03\right)$ and a sex $\times$ stress interaction $\left(\mathrm{F}_{(2,31)}=7, P<0.003\right)$, but without effect of stress $\left(\mathrm{F}_{(2,31)}=1, P>0.05\right)$. However, post-hoc analysis did not reveal any difference between the experimental groups $(P>0.05)$ (Fig. $3 C$ ).

\subsubsection{Adrenals weight}

Analysis of relative adrenals weight (adrenals weight/body weight) indicated a main effect of sex $\left(\mathrm{F}_{(1,31)}=26, P<0.0001\right)$, but without influence of stress $\left(\mathrm{F}_{(2,31)}=2, P>0.05\right)$ and a sex $\times$ stress interaction $\left(\mathrm{F}_{(2,31)}=2, P>0.05\right)$ (Fig. 3C). Post-hoc analysis revealed reduced values in females of control $(P<0.05)$ and RRS $(P<0.05)$ groups, when compared with the respective male groups. Moreover, CVS increased values in males $(P<0.05)$ (Fig. 3C).

\subsubsection{Thymus weight}

Analysis of relative thymus weight (thymus weight/body weight) indicated main effect of $\operatorname{sex}\left(\mathrm{F}_{(1,31)}=6, P<0.01\right)$, but without effect of stress $\left(\mathrm{F}_{(2,31)}=1, P>0.05\right)$ and a sex $\times$ stress interaction $\left(\mathrm{F}_{(2,31)}=1, P>0.05\right)$. However, post-hoc analysis did not reveal any difference between the experimental groups $(P>0.05)$ (Fig. $3 C)$.
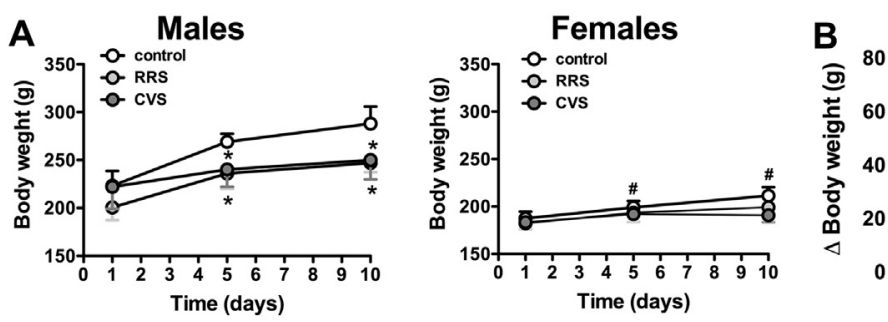

Fig. 3. Body, heart, adrenals, and thymus weights and plasma corticosterone concentration in male and female rats control (white) and subjected to RRS (light grey) or CVS (dark grey). (A) Time-course of body weight. The circles represent the mean \pm SEM. ${ }^{*} P<0.05$ vs respective control group, $\# P<0.05$ vs respective male group. Threeway ANOVA followed by Bonferroni post-hoc test ( $n=6-7$ ) group). (B) Body weight change ( $\Delta$ body weight) from the first to 10th day of chronic stress protocols. The bars represent the mean \pm SEM. ${ }^{*} P<0.05$ vs respective control group, $\# P<0.05$ vs respective male group. Two-way ANOVA followed by Bonferroni post-hoc test $(n=6-7$ / group). (C) Relative weight (organ weigh/body weight) of heart, adrenal, and thymus; and plasma corticosterone concentration. The bars represent the mean \pm SEM. ${ }^{*} P<0.05$ vs respective control group, \#P<0.05 vs respective male group. Two-way ANOVA followed by Bonferroni post-hoc test ( $n=6-7 /$ group).
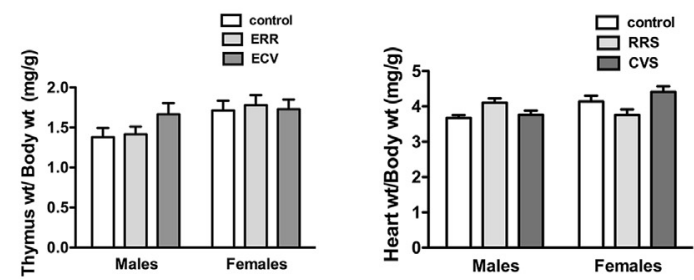

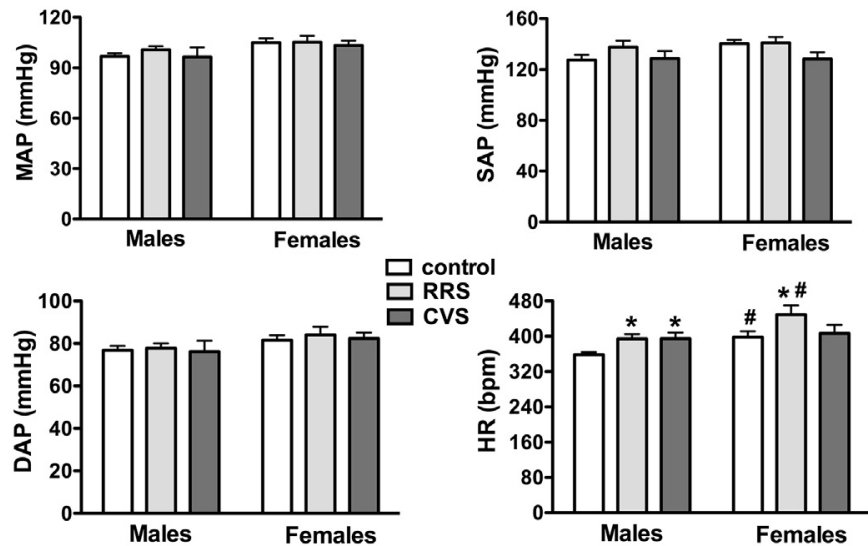

Fig. 4. MAP, SAP, DAP and HR in male and female rats control (white bars) and subjected to RRS (light grey bars) or CVS (dark grey bars). The bars represent the mean \pm SEM. ${ }^{*} P<0.05$ vs respective control group, $\# P<0.05$ vs respective male group. Two-way ANOVA followed by Bonferroni post-hoc test ( $n=6-7$ /group).

\subsubsection{Plasma corticosterone}

Analysis of basal plasma corticosterone concentration did not indicate significant effect of either sex $\left(\mathrm{F}_{(1,31)}=3, P>0.05\right)$ or stress $\left(\mathrm{F}_{(2,31)}=2, P>0.05\right)$ or a gender $\times$ stress interaction $\left(\mathrm{F}_{(2,31)}=2\right.$, $P>0.005$ ) (Fig. 3C). However, post-hoc analysis revealed that RRS $(P<0.05)$ and CVS $(P<0.01)$ increased plasma corticosterone concentration in females (Fig. 3C).

\subsection{Effects of CVS and RRS in female and male rats on basal cardiovascular parameters}

Analysis of MAP and DAP indicated a main effect of sex (MAP: $\mathrm{F}_{(1,31)}=6, P<0.02$; DAP: $\mathrm{F}_{(1,31)}=4, P<0.04$ ), but without effect of stress $\operatorname{(MAP:F}_{(2,31)}=0.4, P>0.05 ;$ DAP: $\mathrm{F}_{(2,31)}=0.2$, $P>0.05)$ and a sex $\times$ stress interaction $\left(\operatorname{MAP}_{(2,31)}=0.2\right.$, $P>0.05$; DAP: $\left.\mathrm{F}_{(2,31)}=0.1, P>0.05\right)$. Analysis of SAP did not identify effect of either sex $\left(\mathrm{F}_{(1,31)}=2, P>0.05\right)$ or stress $\left(\mathrm{F}_{(2,31)}=2, P>0.05\right)$ or a sex $\times$ stress interaction $\left(\mathrm{F}_{(2,31)}=1\right.$, $P>0.05)$. Post-hoc analysis did not reveal any difference between the experimental groups in either MAP $(P>0.05)$, SAP $(P>0.05)$, or DAP $(P>0.05)$ (Fig. 4).

Analysis of basal HR values indicated a main effect of sex $\left(\mathrm{F}_{(1,31)}=8, \quad P<0.009\right)$ and stress $\left(\mathrm{F}_{(2,31)}=4, P<0.03\right)$, but without a sex $\times$ stress interaction $\left(\mathrm{F}_{(2,31)}=1, P>0.05\right)$ (Fig. 4). Posthoc analysis revealed higher values in females of control $(P<0.05)$ and RRS $(P<0.05)$ groups, when compared with the respective male groups (Fig. 4). Furthermore, RRS increased HR in both genders (female: $P<0.05$, male: $P<0.05$ ), whereas CVS evoked resting tachycardia only in males $(P<0.05)$ (Fig. 4).

\subsection{Effects of CVS and RRS in female and male rats on intrinsic HR and cardiac autonomic activity}

\subsubsection{Intrinsic $H R$}

Analysis of the HR values after combined treatment with propranolol and methylatropine (iHR) did not indicate effect of either stress $\left(\mathrm{F}_{(2,31)}=0.3, P>0.05\right)$ or $\operatorname{sex}\left(\mathrm{F}_{(1,31)}=2, P>0.05\right)$ or a sex $\times \mathrm{s}-$ tress interaction $\left(\mathrm{F}_{(2,31)}=0.1, P>0.05\right)($ Fig. 5).

\subsubsection{Cardiac sympathetic activity}

Analysis of the HR change induced by intravenous administration of propranolol indicated a main effect of sex $\left(\mathrm{F}_{(1,31)}=9, P<0.005\right)$ and stress $\left(\mathrm{F}_{(2,31)}=4, P<0.04\right)$, but without a sex $\times$ stress interaction $\left(\mathrm{F}_{(2,31)}=1, P>0.05\right)$ (Fig. 5). Post-hoc analysis revealed increased responses in females of control $(P<0.05)$ and RRS $(P<0.05)$ groups, when compared with the respective male groups (Fig. 5). Furthermore, both RRS $(P<0.001)$ and CVS $(P<0.001)$ increased the propranolol response in males (Fig. 5).

\subsubsection{Cardiac parasympathetic activity}

Analysis of the HR change induced by intravenous administration of methylatropine did not indicate effect of either $\operatorname{sex}\left(\mathrm{F}_{(1,31)}=1\right.$, $P>0.05)$ or stress $\left(\mathrm{F}_{(2,31)}=2, P>0.05\right)$ or a sex $\times$ stress interaction $\left(\mathrm{F}_{(2,31)}=2, P>0.05\right)$ (Fig. 5). However, post-hoc analysis revealed that response in females of control group was reduced when compared with the respective male group $(P<0.05)$ (Fig. 5).

\subsection{Effects of CVS and RRS in female and male rats on baroreflex activity}

Results of baroreflex activity analysis are presented in Fig. 6. Nonlinear regression analysis of baroreflex activity indicated increased upper plateau $\left(\mathrm{P}_{2}\right)$ in female of control group in relation to respective male group $(P<0.01)$. Both RRS $(P<0.01)$ and CVS $(P<0.01)$ reduced the lower plateau $\left(\mathrm{P}_{1}\right)$ in male animals. Additionally, CVS decreased the gain $(\mathrm{G})$ in males $(P<0.01)$.

Linear regression analysis was used for individual analysis of baroreflex function during the pressor and depressor effects of the blood pressure. Analysis indicated that the slope of the regression line for both decreases in HR as response to MAP increase $(P<0.01)$ and tachycardia evoked by MAP decrease $(P<0.01)$ were higher in females of control group, when compared with the respective male group. Additionally, CVS increased reflex tachycardia $(P<0.05)$ in males.

\subsection{Effects of CVS and RRS in female and male rats on blood pressure response to vasoactive agents}

Results of MAP changes evoked by intravenous infusion of vasoactive agents are presented in Fig. 7 and Table 1.

\subsubsection{Phenylephrine}

Analysis of the pressor response caused by intravenous infusion of the vasoconstrictor agent phenylephrine indicated that CVS increased the $\mathrm{E}_{\max }(P<0.05)$ in males.

\subsubsection{Acetylcholine}

Analysis of the depressor response to intravenous infusion of the vasodilator agent acetylcholine indicated that the CVS $(P<0.01)$ reduced the $E_{\max }$ of the dose-response curve in male animals. Furthermore, both RRS $(P<0.01)$ and CVS $(P<0.001)$ increased $E_{\max }$ in females.

\subsubsection{Sodium nitroprusside}

Analysis of the depressor response to intravenous infusion of the vasodilator agent SNP indicated that RRS decreased $\mathrm{ED}_{50}(P<0.01)$ in males. Additionally, both RRS $(P<0.01)$ and CVS $(P<0.05)$ decreased $\mathrm{E}_{\max }$ in females.

\section{Discussion}

This study is the first to investigate the influence of sexual dimorphisms on impact of CVS and RRS on cardiovascular function and autonomic activity, as well as to evaluate the influence of predictability of the stressor stimulus on its effects in female animals. Present results are also the first to evaluate an interplay between sex and different chronic stress regimens on somatic, neuroendocrine and behavioral responses to stress. Table 2 summarizes the main findings documented in the present study. 
A Propranolol (sympathetic activity)

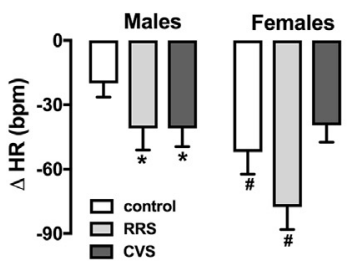

B Methylatropine (parasympathetic activity)

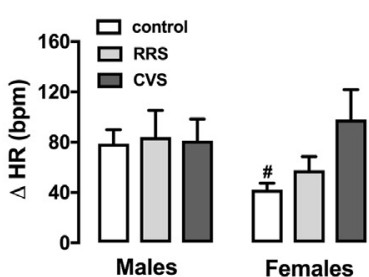

C Propranolol + Methylatropine (intrinsic HR)

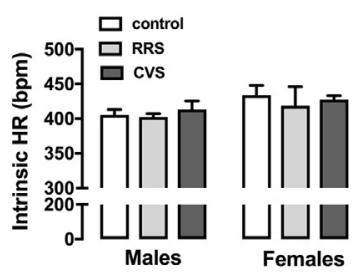

Fig. 5. Cardiac autonomic activity and intrinsic HR in male and female rats control (white) and subjected to RRS (light grey) or CVS (dark grey). (A) HR change $(\Delta \mathrm{HR})$ evoked by systemic administration of the $\beta$-adrenoceptor antagonist propranolol (sympathetic activity). (B) $\Delta$ HR evoked by intravenous administration of the muscarinic receptor antagonist methylatropine (parasympathetic activity). (C) HR values after combined treatment with methylatropine and propranolol (intrinsic HR). The bars represent the mean \pm SEM. ${ }^{*} P<0.05$ vs respective control group, ${ }^{\#} P<0.05$ vs respective male group. Two-way ANOVA followed by Bonferroni post-hoc test ( $n=6-7 /$ group).

\section{A $\quad$ Males}
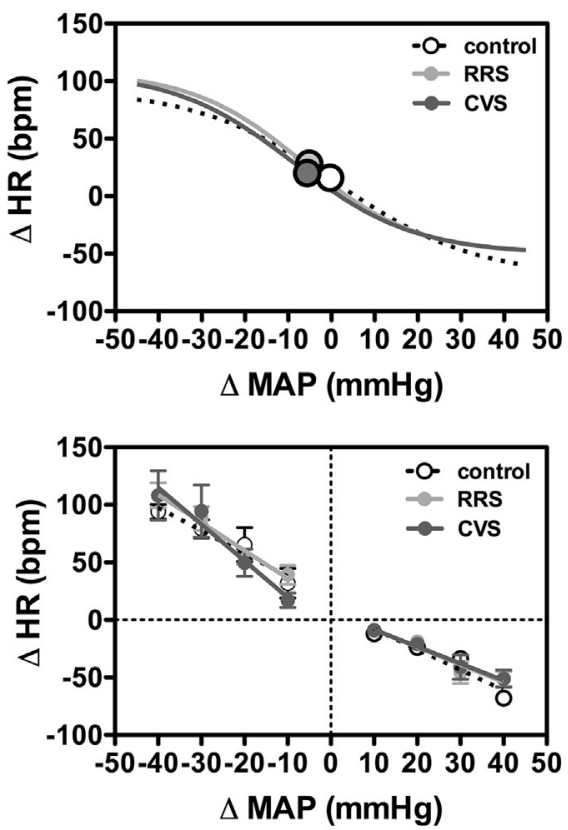

Females

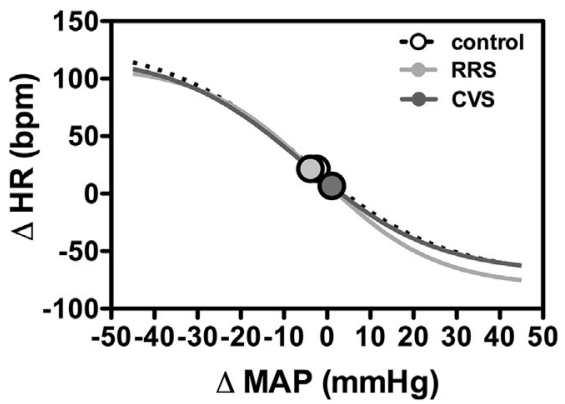

Fig. 6. Analysis of baroreflex activity in male and female rats control (white) and subjected to RRS (light grey) or CVS (dark grey). (A) Non-linear (top) and linear (bottom) regression analysis of the baroreflex correlating mean arterial pressure change ( $\triangle \mathrm{MAP}$ ) evoked by intravenous infusion of phenylephrine and SNP and the reflex HR response $(\Delta \mathrm{HR})$. Symbols on sigmoid curves indicate the $\mathrm{BP}_{50}(n=6-7 /$ group $)$. (B) Parameters derived from nonlinear $\left(\mathrm{G}, \mathrm{P}_{1}, \mathrm{P}_{2}\right.$, HR range and $\left.\mathrm{BP}_{50}\right)$ and linear (slope bradycardia and slope tachycardia) regression analysis of the baroreflex. The bars represent the mean \pm SEM. ${ }^{*} P<0.05$ vs respective control group, ${ }^{\#} P<0.05$ vs respective male group. Two-way ANOVA followed by Bonferroni post-hoc test ( $n=6-7 /$ group).
B

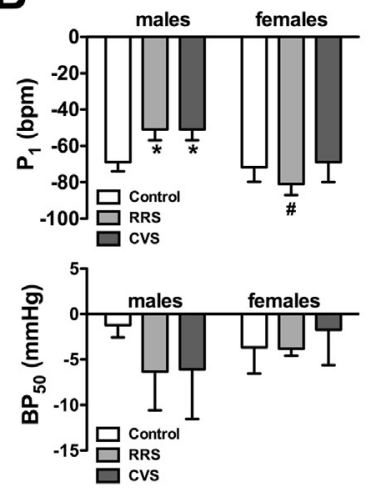

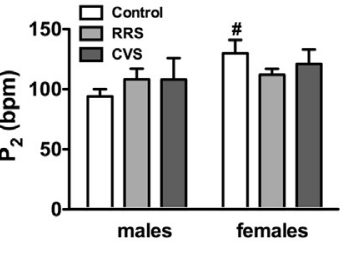
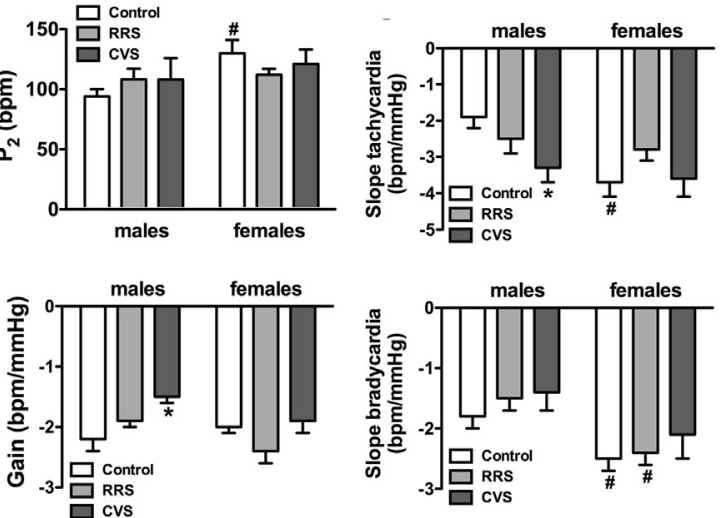

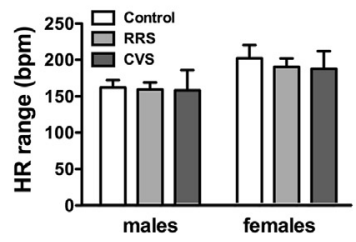

4.1. Stress-evoked behavioral, somatic and neuroendocrine changes: influence of sex and stress type

Studies have demonstrated that the reduction in body weight gain evoked by either CVS or RRS is absent or lesser in females relative to males (Dalla et al., 2005; Doremus-Fitzwater et al., 2009; Duncko et al., 2001; Xing et al., 2013; Xu et al., 2010), thus supporting the present findings. Accordingly, the stress-evoked reduction of food intake is lesser in females than males (Lenglos et al., 2013). Glucocorticoids stimulate food intake and may modulate the feeding response to stress 

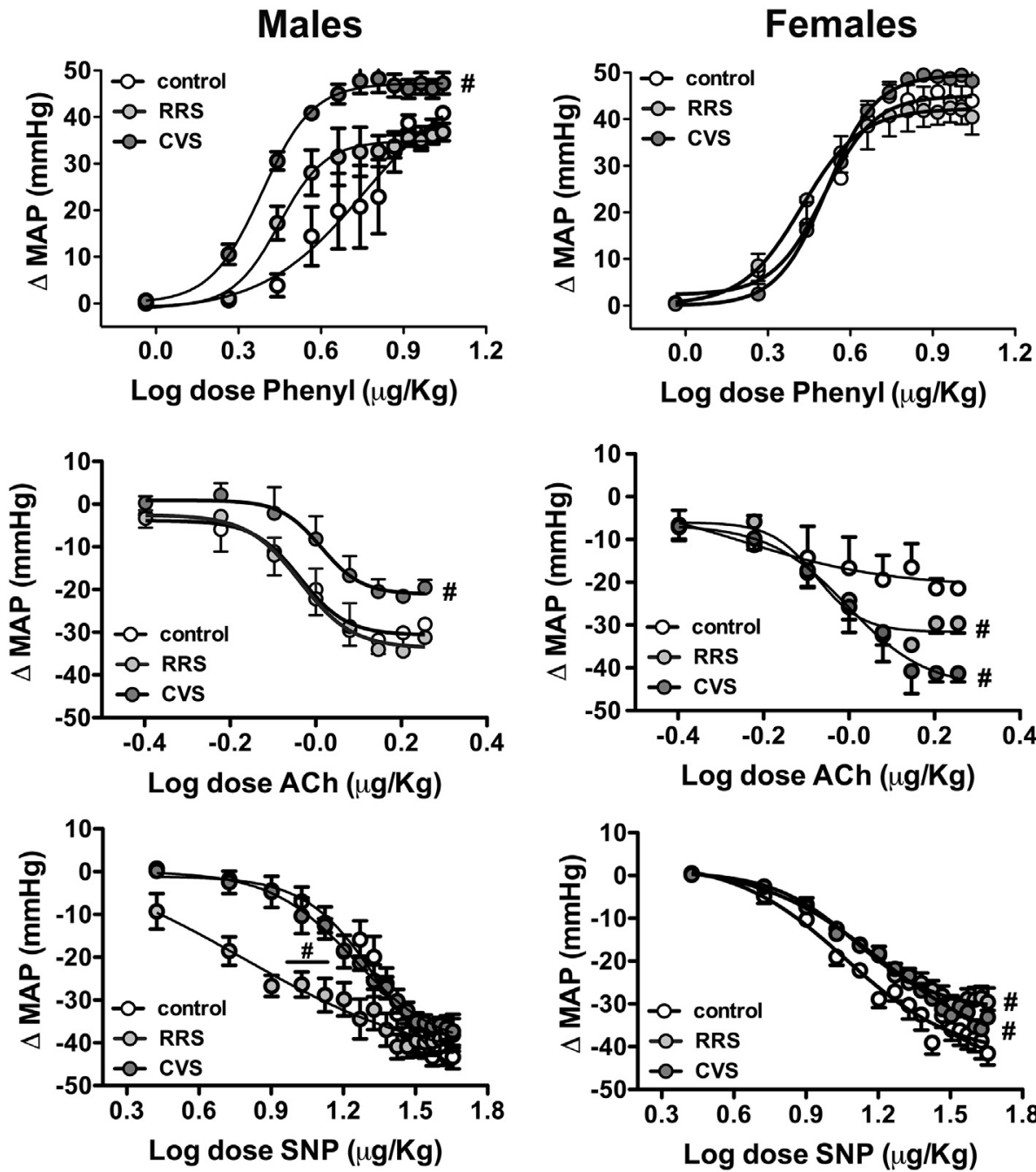
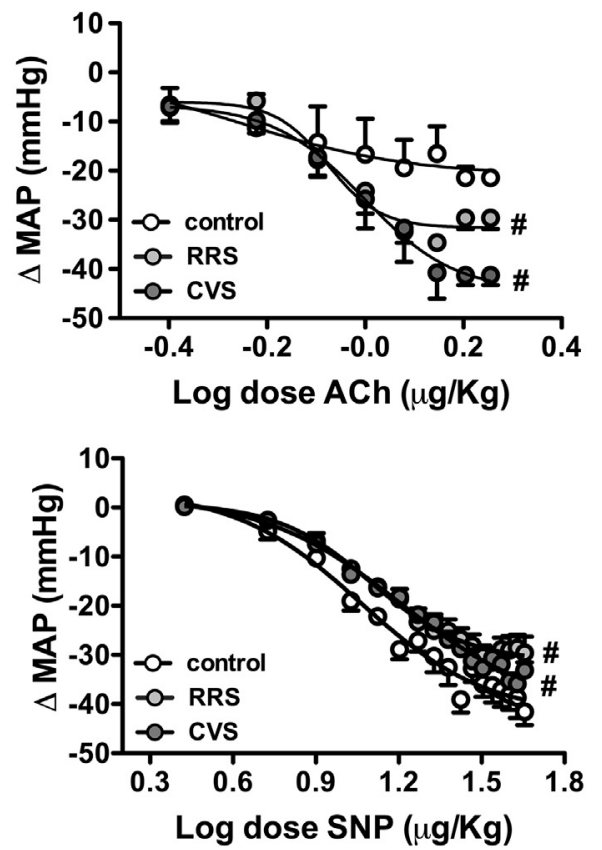

Fig. 7. Mean arterial pressure change ( $\triangle \mathrm{MAP}$ ) evoked by increasing concentrations of phenylephrine (Phenyl), acetylcholine (Ach), and SNP in male (left) and female (right) rats control (white circles) and subjected to RRS (light grey circles) or CVS (dark grey circles). The circles represent the mean \pm SEM. ${ }^{\#} P<0.05$ vs respective control group (see parameters in Table 1) ( $n=6-7 /$ group $)$. (la Fleur, 2006). Therefore, the increased corticosterone concentration evoked by both RRS and CVS in females (see discussion below) could buffer the anorexic effect of these stressors, which in turn reduces the impact on body weight gain. It is also important to note that the body weight gain is decreased in females, which is due to influence of estrogens on energy homeostasis (e.g., food intake inhibition, increase in insulin sensitivity, and thermogenesis) (Lopez and Tena-Sempere, 2015). Previous studies indicated that chronic stressors may decrease plasma estradiol levels in female rats (Galea et al., 1997), so that effect of stress may also be counterbalanced by a reduced influence of estrogens on energy homeostasis in females.

The adrenal hypertrophy evoked by CVS selectively in males in the

Table 1

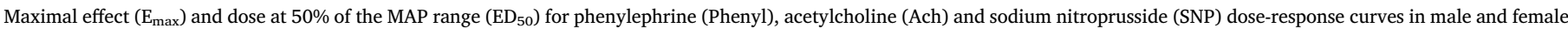
animals subjected to repeated restraint stress (RRS) or chronic variable stress (CVS) for 10 consecutive days.

\begin{tabular}{|c|c|c|c|c|c|c|}
\hline \multirow[t]{2}{*}{ Group } & \multicolumn{2}{|l|}{ Phenyl } & \multicolumn{2}{|l|}{ Ach } & \multicolumn{2}{|l|}{ SNP } \\
\hline & $\mathrm{ED}_{50}(\log$ dose$)$ & $\mathrm{E}_{\max }(\mathrm{mmHg})$ & $\mathrm{ED}_{50}(\log$ dose $)$ & $\mathrm{E}_{\max }(\mathrm{mmHg})$ & $\mathrm{ED}_{50}(\log$ dose $)$ & $\mathrm{E}_{\max }(\mathrm{mmHg})$ \\
\hline \multicolumn{7}{|l|}{ Males } \\
\hline Control & $0.68 \pm 0.07$ & $40 \pm 1$ & $-0.08 \pm 0.06$ & $-29 \pm 3$ & $1.3 \pm 0.05$ & $-43 \pm 3$ \\
\hline RRS & $0.51 \pm 0.07$ & $37 \pm 2$ & $-0.05 \pm 0.2$ & $-31 \pm 1$ & $0.5 \pm 0.1^{*}$ & $-38 \pm 3$ \\
\hline CVS & $0.45 \pm 0.09$ & $48 \pm 2$ & $0.04 \pm 0.07$ & $-19 \pm 2$ & $1.2 \pm 0.03$ & $-37 \pm 4$ \\
\hline \multicolumn{7}{|l|}{ Females } \\
\hline Control & $0.47 \pm 0.05$ & $44 \pm 3$ & $0.04 \pm 0.1$ & $-21 \pm 1$ & $1.0 \pm 0.04$ & $-42 \pm 2$ \\
\hline RRS & $0.41 \pm 0.04$ & $41 \pm 4$ & $-0.11 \pm 0.09$ & $-31 \pm 2$ & $1.1 \pm 0.04^{\#}$ & $-29 \pm 3^{*}$ \\
\hline CVS & $0.49 \pm 0.05$ & $47 \pm 2$ & $0.01 \pm 0.09$ & $-42 \pm 2^{*}$ & $1.2 \pm 0.05$ & $-33 \pm 1$ \\
\hline Interaction & $F_{(2,31)}=1.7 P^{>} 0.05$ & $F_{(2,31)}=0.5 P^{>} 0.05$ & $F_{(2,31)}=0.4 P>0.05$ & $F_{(2,31)}=29 P<0.0001$ & $F_{(2,31)}=15 P<0.0001$ & $F_{(2,31)}=1 P^{>} 0.05$ \\
\hline Gender & $F_{(2,31)}=2.3 P^{>} 0.05$ & $F_{(1,31)}=1 P^{>} 0.05$ & $F_{(1,31)}=0.01 P^{>} 0.05$ & $F_{(1,31)}=8 P^{<} 0.009$ & $F_{(1,31)}=3 P^{>} 0.05$ & $F_{(1,30)}=4 P<0.05$ \\
\hline Stress & $F_{(1,31)}=1.8 P>0.05$ & $F_{(2,31)}=6 P<0.007$ & $F_{(2,31)}=0.5 P^{>} 0.05$ & $F_{(2,31)}=5 P^{<} 0.01$ & $F_{(2,31)}=15 P<0.0001$ & $F_{(2,31)}=6 P<0.009$ \\
\hline
\end{tabular}

Two-way ANOVA followed by Bonferroni post hoc test.

Values are mean \pm SEM.

${ }^{*} P<0.05$ vs control group within same gender.

${ }^{\#} P<0.05$ vs respective male group. 
Table 2

Summary of the effects of chronic stress regimens in male and female rats.

\begin{tabular}{|c|c|c|c|c|}
\hline & \multicolumn{2}{|c|}{ Male } & \multicolumn{2}{|c|}{ Female } \\
\hline & RRS & CVS & RRS & CVS \\
\hline \multicolumn{5}{|l|}{ Behavior } \\
\hline Anxiety-like behaviors ${ }^{\mathrm{b}}$ & - & - & $\uparrow$ & $\uparrow$ \\
\hline \multicolumn{5}{|l|}{ Somatic parameters } \\
\hline Body weight $t^{\mathrm{b}}$ & $\downarrow$ & $\downarrow$ & - & - \\
\hline Heart weight & - & - & - & - \\
\hline Adrenal weight & - & $\uparrow$ & - & - \\
\hline Thymus weight & - & - & - & - \\
\hline \multicolumn{5}{|l|}{ HPA axis } \\
\hline Corticosterone & - & - & $\uparrow$ & $\uparrow$ \\
\hline \multicolumn{5}{|l|}{ Cardiovascular baseline } \\
\hline Arterial pressure & - & - & - & - \\
\hline$H R^{\mathrm{b}}$ & $\uparrow$ & $\uparrow$ & $\uparrow$ & - \\
\hline \multicolumn{5}{|l|}{ Cardiac autonomic activity } \\
\hline Sympathetic activity & $\uparrow$ & $\uparrow$ & $\uparrow^{\mathrm{a}}$ & - \\
\hline Vagal activity ${ }^{\mathrm{b}}$ & - & - & - & - \\
\hline Intrinsic HR & - & - & - & - \\
\hline Baroreflex bradycardia $^{\mathrm{b}}$ & $\downarrow$ & $\downarrow$ & - & - \\
\hline Baroreflex tachycardia $^{\mathrm{b}}$ & - & $\uparrow$ & - & - \\
\hline \multicolumn{5}{|l|}{ Vascular function } \\
\hline Pressor response to phenylephrine & - & $\uparrow$ & - & - \\
\hline Depressor effect of acetylcholine & - & $\downarrow$ & $\uparrow$ & $\uparrow$ \\
\hline Depressor effect of sodium nitroprusside & $\uparrow$ & - & $\downarrow$ & $\downarrow$ \\
\hline
\end{tabular}

Up or down arrows indicate significant increase or decrease, respectively.

a Statistically nonsignificant but relevant (increase of 48\%).

${ }^{\mathrm{b}}$ Sex differences in control groups.

present study is in line with previous evidence that males are more susceptible to adrenal hypertrophy and thymus involution caused by chronic stressors (Toth et al., 2008; Xu et al., 2010). Conversely, we observed that both stress protocols increased plasma corticosterone concentration only in females. Our results corroborate previous studies reporting increases in basal concentration of plasma corticosterone in females but not male animals following CVS (Dalla et al., 2005; Xing et al., 2013). Habituation of the HPA axis response normally observed upon repeated exposure to the same stressor is reduced or slower in females (Bhatnagar et al., 2005; Chadda and Devaud, 2005; DoremusFitzwater et al., 2009; Galea et al., 1997). The reduced habituation process may explain the corticosterone response to RRS in females, since it has been proposed that it constitutes an important adaptive mechanism minimizing the impact of stress (Crestani, 2016; Herman, 2013; McCarty, 2016). Furthermore, decreased negative feedback regulation of HPA axis (Xing et al., 2013) and HPA axis hyperresponsiveness during aversive threats (Doremus-Fitzwater et al., 2009; Galea et al., 1997; Handa et al., 1994; Iwasaki-Sekino et al., 2009; Mitsushima et al., 2003; Rivier, 1999) may also account to plasma corticosterone increase evoked by chronic stressors in females.

Present findings also indicated a vulnerability of females to anxiogenic effects of chronic stressors. Our data are in line with previous evidence of anxiogenic-like effect in the EPM and OF only in females following exposure to either RRS or CVS (Huynh et al., 2011; Lu et al., 2015) However, contrasting data have been reported regarding sexual dimorphisms in impact of CVS and RRS on anxiety-like behaviors. For instance, it was reported anxiogenic-like effects in the EPM selectively in male animals following RRS (Bowman et al., 2009; Noschang et al., 2009). Differences in stress protocol may explain the discrepancy with present findings, since either the duration of sessions ( $6 \mathrm{~h}$ vs $1 \mathrm{~h}$ ) (Bowman et al., 2009) or the protocol length (50 days vs 10 days) (Noschang et al., 2009) were greater than that of the present study. Nevertheless, it was also reported similar decrease in exploration of EPM open arms in male and female following ECV (Dalla et al., 2005), as well as absence of changes in behaviors in the OP in animals exposed to RRS (Albonetti and Farabollini, 1992; Noschang et al., 2009; PerrotSinal et al., 2004).
Regarding the stress type-influence on neuroendocrine and behavioral responses to stress, studies in male rodents have demonstrated that adrenal hypertrophy, thymic involution, changes in HPA axis activity, and increased anxiety- and depression-like behaviors are mainly observed after exposure to CVS protocols, whereas RRS minimally affects these parameters (Haile et al., 2001; Magarinos and McEwen, 1995; Marin et al., 2007; Pastor-Ciurana et al., 2014). The lesser impact of homotypic stressors is possibly related to a habituation process upon repeated exposure to the same stressor, which minimize the impact of stress (Crestani, 2016; Herman, 2013; McCarty, 2016). The increased adrenal weight evoked selectively by CVS in males in the present study is in line with these pieces of evidence. However, none of the stress protocols affected the thymus weight and plasma corticosterone concentration in male animals. Chronic stress-evoked changes in basal corticosterone concentration are inconsistent, and our results corroborate previous evidence of absence of effect following exposure to either RRS or CVS (Duarte et al., 2015; Helmreich et al., 1997; Radley and Sawchenko, 2015). Thymus was demonstrated to be more affected by physical stressors than by psychological aversive stimuli (Isgor et al,, 2004), which may explain our findings. Nevertheless, results of the present study corroborate reports that RRS and CVS similarly reduce body weight gain (Duarte et al., 2015; Magarinos and McEwen, 1995; Vyas et al., 2002).

Interestingly, a stress type-specific effect in somatic parameters, neuroendocrine function and anxiety-like behaviors was not evidenced in female animals in the present study. Indeed, both stressors increased basal plasma corticosterone concentration and anxiety-like behaviors, without affecting body, adrenals, and thymus weights. These results indicate that a vulnerability of females to stress-evoked HPA axis changes and anxiogenic effects are independent of the predictability of the stressor stimulus.

\subsection{Stress-evoked cardiovascular changes: influence of sex and stress type}

Previous studies demonstrated greater increase in sympathetic activity, blood pressure, and HR during acute aversive situations in female than male animals (Anishchenko et al., 2007; Azar et al., 2005; Eikelis and Van Den Buuse, 2000; Weinstock et al., 1998). However, to the best of our knowledge, results from this study are the first to show enduring effect of CVS and RRS on cardiovascular function and autonomic activity in female animals. Our results suggest gender-specific effects of chronic stressors in autonomic activity and cardiovascular function. For instance, CVS caused a sympathetically-mediated increase on HR in males but not female animals. These data corroborate recent results from Stanley et al. (2014) who reported that CVS-evoked endothelial dysfunction in aorta and skeletal muscle arterioles were less pronounced in female than male mice. Nevertheless, RRS increased cardiac sympathetic activity and HR in female animals, an effect also observed in males. RRS decreases plasma estradiol levels in female rats (Galea et al., 1997).

Regarding the influence of stress type, a recent study comparing RRS and CVS in male rats demonstrated that only the latter evoked a sympathetically-mediated increase in basal HR and impaired the baroreflex function (Duarte et al., 2015). These findings contrast present results demonstrating that both chronic stressors similarly affected HR, cardiac sympathetic activity and baroreflex function in male rats. As stated above, adaptation to chronic stress has been proposed to be determined by habituation of physiological responses upon repeated exposure to the same stressor, which limits its long-term impact (Crestani, 2016; Herman, 2013; McCarty, 2016). However, contrary to the well established habituation of HPA axis response (Grissom and Bhatnagar, 2009), cardiovascular responses seems not habituate upon repeated exposure to the same stressor (for review see (Crestani, 2016), thus indicating a reduced process of adaptation. It supports the present findings, as well as previous studies reporting significant cardiovascular dysfunctions and autonomic imbalance following RRS exposure 
(Daubert et al., 2012; Habib et al., 2015; Yang et al., 2014).

Interestingly, our results suggest that overall females are more sensitive to cardiovascular and autonomic effects of RRS than those evoked by CVS. As stated above, previous studies have reported that restraint stress decrease estradiol levels and its effects (Galea et al., 1997; Solomon and Herman, 2009; Walf and Frye, 2005), thus indicating that a decreased cardiovascular protective effects of estrogens may mediate RRS-evoked cardiovascular and autonomic changes. Reduction in estradiol levels following CVS in females was also reported (Lu et al., 2015). However, the reduced cardiovascular and autonomic changes observed in the present study indicates that this effect is likely counterbalanced by changes in estrogen signaling (at least in cardiovascular tissues) and/or activation of other cardiovascular protective mechanisms, which minimize the impact of CVS in cardiovascular function. Anyway, the mechanisms involved in susceptibility and resilience to RRS- and CVS-evoked cardiovascular changes, respectively, in females deserve further investigation.

\subsection{Mechanisms of stress-evoked cardiovascular changes}

Impairment of baroreflex function following either RRS or CVS corroborates previous reports (Almeida et al., 2015; Costa-Ferreira et al., 2016; Duarte et al., 2015; Porter et al., 2004). However, protocol of CVS longer (4 weeks) than that employed in the present study did not affect baroreflex control of HR (Grippo et al., 2008), whereas shorter RRS protocol (5 days) facilitated baroreflex bradycardia (Conti et al., 2001). Indeed, effects of chronic stressors on baroreflex function seem to be related to chronic stress length (Crestani, 2016).

Impairment of baroreflex activity has been related to an increase of sympathetic activity (Grassi et al., 2006). Therefore, the decreased reflex bradycardia in males following both RRS and CVS suggests an involvement of baroreflex changes in the sympathetically-mediated resting tachycardia evoked by these stressors. Interestingly, none of the chronic stressors affected the baroreflex function in females, thus suggesting sex-specific mechanisms mediating the cardiovascular and autonomic changes to stress. Therefore, the RRS-evoked increase in cardiac sympathetic activity that in turn evoked resting tachycardia seems to be mediated by central mechanisms acting independently of baroreflex in female animals. It has been proposed that cardiovascular protective effects of ovarian hormones in females is mediated, at least in part, by it action in brain regions controlling autonomic activity (Hay, 2016). Indeed, a facilitatory influence of ovarian hormones on baroreflex function has been reported (Brooks et al., 2012). Nevertheless, although evidence that chronic stressors decrease estradiol levels and its effects (Galea et al., 1997; Lu et al., 2015; Walf and Frye, 2005), reasons for the lack of effect of chronic stressors in baroreflex function, as well as the mechanisms related to RRS-evoked cardiovascular and autonomic changes in females deserve further investigation.

Our results in males corroborate previous in vitro and in vivo results demonstrating decreased vascular reactivity to a variety of cholinergic agonists following CVS (Almeida et al., 2015; Bayramgurler et al., 2013; Isingrini et al., 2012; Stanley et al., 2014). The enhanced phenylephrine response is also in line with report of enhanced responsiveness of saphenous and mesenteric arteries of mice subjected to CVS to noradrenaline (Bouzinova et al., 2012). Evidence of the impact of chronic stressors in vascular function in females is limited. A recent study reported impairment of vascular reactivity to cholinergic agonists in conduit arteries and skeletal muscle arterioles following CVS in female mice (Stanley et al., 2014), which contrast with the facilitation of blood pressure response to acetylcholine observed in the present study. Present study is the first to investigate the impact of chronic stressors in responsiveness to vasoactive agents using an in vivo approach, which may explain the discrepancy with previous studies. In this sense, although the blood pressure changes evoked by all vasoactive agents to be clearly related to a vascular action, we cannot to exclude the possibility of influence of drug action on other sites, which is peculiar of in vivo treatments. It is especially clear for acetylcholine, once this drug can act on autonomic ganglion and heart in addition to vascular wall. Nevertheless, specie differences (mice vs rats) may also account to divergence. Furthermore, our findings are supported by in vitro evidence that restraint stress facilitate endothelial production of nitric oxide (Cordellini and Vassilieff, 1998; Junior and Cordellini, 2007).

Interestingly, the facilitation of the depressor response to acetylcholine was followed by decreased responsiveness to NPS in females following either RRS or CVS. Taken together, these data indicate that an improvement in endothelial function is likely followed by impairment in nitric oxide signaling in vascular wall. However, the facilitation of acetylcholine response indicates that the improvement in endothelial function seems to overlap the changes in nitric oxide signaling. In this sense, recruitment of other relaxing factors released from endothelium to compensate impairment in nitric oxide-dependent relaxation was reported in animals subjected to chronic stress (Isingrini et al., 2012).

Increased vascular tone in hypertension has been associated with endothelial dysfunction and decreased dilatation of blood vessels (Tang and Vanhoutte, 2010). Increased vascular reactivity to vasoconstrictor agents has also been related to hypertension (Chang et al., 2002; Laurent et al., 1987). Therefore, the increase in response to acetylcholine in females evoked by RRS and CVS as well as the facilitation of NPS response in males subjected to RRS may constitute important adaptive responses counteracting changes that contribute to blood pressure rise. The reduction in acetylcholine response and facilitation of pressor response to phenylephrine in male animals subjected to CVS contribute to an increase in blood pressure. However, the lack of blood pressure changes indicates that these effects are likely buffered by other compensatory responses.

Despite the importance of estrogens in the development of behavioral and physiological changes evoked by stress (Kudielka and Kirschbaum, 2005; Maeng and Milad, 2015; Solomon and Herman, 2009), we have not considered the estrous cycle of the animals in the present study. In this sense, the aim in this investigation was not to test the effect of cyclic variation in behavioral, somatic and physiological parameters. As such, results reported in the present study rather provide evidence of differences that are associated with the female sex and remain in place throughout the estrous cycle, independently of fluctuations in hormonal profiles. Nevertheless, future experiments considering the different cycle phases are important for a better understanding of stress impacts in females.

In summary, present findings indicate a sex- and stress type-specific influence of chronic stressors. Our results suggest that regardless of stress type, males are more vulnerable to somatic effects, while females appear to be more susceptible to changes in HPA axis activity and anxiogenic effects evoked by chronic stressors. Stress type-specific influence was evidenced on cardiovascular function and autonomic activity in females, as changes in these animals were evoked only by the RRS. However, both stressors similarly affected these parameters in male animals. Impairment of baroreflex function seem to be an important mechanism mediating the stress-evoked cardiovascular/autonomic changes evoked by both stressors in males, while females are resilient to effects of chronic stressors on this reflex cardiovascular mechanism. Finally, our results indicate that effects of stress in responsiveness to vasoactive agents are also sex-specific. In this sense, changes in females seem to constitute adaptive responses counteracting changes that contribute to blood pressure rise, whereas some effects in males are likely buffered by other compensatory responses that avoid blood pressure rise.

\section{Acknowledgments}

The authors wish to thank Elisabete Lepera and Rosana Silva for technical assistance. This work was supported by FAPESP grant \# 2012/14376-0 and 2015/05922-9, CNPq grant \# 456405/2014-3, and Programa de Apoio ao Desenvolvimento Científico da Faculdade de 


\section{Ciências Farmacêuticas da UNESP - PADC. CCC is a CNPq research fellow (process \# 305583/2015-8).}

\section{References}

Albonetti, M.E., Farabollini, F., 1992. Behavioural responses to single and repeated restraint in male and female rats. Behav. Process. 28 (1-2), 97-109.

Almeida, J., Duarte, J.O., Oliveira, L.A., Crestani, C.C., 2015. Effects of nitric oxide synthesis inhibitor or fluoxetine treatment on depression-like state and cardiovascular changes induced by chronic variable stress in rats. Stress 18 (4), 462-474.

Anishchenko, T.G., Glushkovskaya-Semyachkina, O.V., Berdnikova, V.A., Sindyakova, T.A., 2007. Sex-related differences in cardiovascular stress reactivity in healthy and hypertensive rats. Bull. Exp. Biol. Med. 143 (2), 178-181.

Azar, T., Sharp, J., Lawson, D., 2005. Stress-like cardiovascular responses to common procedures in male versus female spontaneously hypertensive rats. Contemp. Top. Lab. Anim. Sci. 44 (3), 25-30.

Bayramgurler, D., Karson, A., Yazir, Y., Celikyurt, I.K., Kurnaz, S., Utkan, T., 2013. The effect of etanercept on aortic nitric oxide-dependent vasorelaxation in an unpredictable chronic, mild stress model of depression in rats. Eur. J. Pharmacol. 710 (1-3), 67-72.

Bekker, M.H., van Mens-Verhulst, J., 2007. Anxiety disorders: sex differences in prevalence, degree, and background, but gender-neutral treatment. Gend. Med. 4 (Suppl B), S178-193.

Bernatova, I., Puzserova, A., Dubovicky, M., 2010. Sex differences in social stress-induced pressor and behavioral responses in normotensive and prehypertensive rats. Gen. Physiol. Biophys. 29 (4), 346-354.

Bhatnagar, S., Lee, T.M., Vining, C., 2005. Prenatal stress differentially affects habituation of corticosterone responses to repeated stress in adult male and female rats. Horm. Behav. 47 (4), 430-438.

Bouzinova, E.V., Moller-Nielsen, N., Boedtkjer, D.B., Broegger, T., Wiborg, O., Aalkjaer, C., Matchkov, V.V., 2012. Chronic mild stress-induced depression-like symptoms in rats and abnormalities in catecholamine uptake in small arteries. Psychosom. Med. 74 (3), 278-287.

Bowman, R.E., Beck, K.D., Luine, V.N., 2003. Chronic stress effects on memory: sex differences in performance and monoaminergic activity. Horm. Behav. 43 (1), 48-59.

Bowman, R.E., Micik, R., Gautreaux, C., Fernandez, L., Luine, V.N., 2009. Sex-dependent changes in anxiety, memory, and monoamines following one week of stress. Physiol. Behav. 97 (1), 21-29.

Brooks, V.L., Cassaglia, P.A., Zhao, D., Goldman, R.K., 2012. Baroreflex function in females: changes with the reproductive cycle and pregnancy. Gend. Med. 9 (2), 61-67.

Burt, V.L., Whelton, P., Roccella, E.J., Brown, C., Cutler, J.A., Higgins, M., Horan, M.J., Labarthe, D., 1995. Prevalence of hypertension in the US adult population. Results from the Third National Health and Nutrition Examination Survey, 1988-1991. Hypertension 25 (3), 305-313.

Carvalho-Netto, E.F., Myers, B., Jones, K., Solomon, M.B., Herman, J.P., 2011. Sex differences in synaptic plasticity in stress-responsive brain regions following chronic variable stress. Physiol. Behav. 104 (2), 242-247.

Chadda, R., Devaud, L.L., 2005. Differential effects of mild repeated restraint stress on behaviors and GABA(A) receptors in male and female rats. Pharmacol. Biochem. Behav. 81 (4), 854-863.

Chang, H.R., Lee, R.P., Wu, C.Y., Chen, H.I., 2002. Nitric oxide in mesenteric vascular reactivity: a comparison between rats with normotension and hypertension. Clin. Exp. Pharmacol. Physiol. 29 (4), 275-280.

Conti, L.H., Shannon, M.H., Murry, J.D., Printz, M.P., 2001. Repeated restraint stressinduced increase in baroreceptor reflex sensitivity: role of corticotropin-releasing factor. Neuropeptides 35 (2), 71-81.

Cordellini, S., Vassilieff, V.S., 1998. Decreased endothelium-dependent vasoconstriction to noradrenaline in acute-stressed rats is potentiated by previous chronic stress: nitric oxide involvement. Gen. Pharmacol. 30 (1), 79-83.

Costa-Ferreira, W., Vieira, J.O., Almeida, J., Gomes-de-Souza, L., Crestani, C.C., 2016. Involvement of type 1 Angiontensin II receptor (AT1) in cardiovascular changes in duced by chronic emotional stress: comparison between homotypic and heterotypic stressors. Front. Pharmacol. 7, 262.

Crestani, C.C., 2016. Emotional stress and cardiovascular complications in animal models: a review of the influence of stress type. Front. Physiol. 7, 251.

Crestani, C.C., Alves, F.H., Busnardo, C., Resstel, L.B., Correa, F.M., 2010. N-methyl-Daspartate glutamate receptors in the hypothalamic paraventricular nucleus modulate cardiac component of the baroreflex in unanesthetized rats. Neurosci. Res. 67 (4), 317-326.

Cruz, F.C., Duarte, J.O., Leao, R.M., Hummel, L.F., Planeta, C.S., Crestani, C.C., 2016. Adolescent vulnerability to cardiovascular consequences of chronic social stress: immediate and long-term effects of social isolation during adolescence. Dev. Neurobiol. 76 (1), 34-46.

Dalla, C., Antoniou, K., Drossopoulou, G., Xagoraris, M., Kokras, N., Sfikakis, A., Papadopoulou-Daifoti, Z., 2005. Chronic mild stress impact: are females more vulnerable? Neuroscience 135 (3), 703-714.

Daubert, D.L., McCowan, M., Erdos, B., Scheuer, D.A., 2012. Nucleus of the solitary tract catecholaminergic neurons modulate the cardiovascular response to psychological stress in rats. J. Physiol. 590 (Pt 19), 4881-4895.

Doremus-Fitzwater, T.L., Varlinskaya, E.I., Spear, L.P., 2009. Social and non-social anxiety in adolescent and adult rats after repeated restraint. Physiol. Behav. 97 (3-4), 484-494.

Duarte, J.O., Cruz, F.C., Leao, R.M., Planeta, C.S., Crestani, C.C., 2015. Stress vulnerability during adolescence: comparison of chronic stressors in adolescent and adult rats. Psychosom. Med. 77 (2), 186-199.

Duncko, R., Kiss, A., Skultetyova, I., Rusnak, M., Jezova, D., 2001. Corticotropin-releasing hormone mRNA levels in response to chronic mild stress rise in male but not in female rats while tyrosine hydroxylase mRNA levels decrease in both sexes. Psychoneuroendocrinology 26 (1), 77-89.

Eikelis, N., Van Den Buuse, M., 2000. Cardiovascular responses to open-field stress in rats: sex differences and effects of gonadal hormones. Stress 3 (4), 319-334.

Engi, S.A., Planeta, C.S., Crestani, C.C., 2016. Effect of voluntary ethanol consumption combined with testosterone treatment on cardiovascular function in rats: Influence of exercise training. PLoS One 11 (1), e0146974.

la Fleur, S.E., 2006. The effects of glucocorticoids on feeding behavior in rats. Physiol. Behav. 89 (1), 110-114.

Galea, L.A., McEwen, B.S., Tanapat, P., Deak, T., Spencer, R.L., Dhabhar, F.S., 1997. Sex differences in dendritic atrophy of CA3 pyramidal neurons in response to chronic restraint stress. Neuroscience 81 (3), 689-697.

Gouveia, M.K., Miguel, T.T., Busnardo, C., Scopinho, A.A., Correa, F.M., Nunes-de-Souza, R.L., Crestani, C.C., 2016. Dissociation in control of physiological and behavioral responses to emotional stress by cholinergic neurotransmission in the bed nucleus of the stria terminalis in rats. Neuropharmacology 101, 379-388.

Grassi, G., Trevano, F.Q., Seravalle, G., Scopelliti, F., Mancia, G., 2006. Baroreflex function in hypertension: consequences for antihypertensive therapy. Prog. Cardiovasc. Dis. 48 (6), 407-415.

Grippo, A.J., Moffitt, J.A., Johnson, A.K., 2008. Evaluation of baroreceptor reflex function in the chronic mild stress rodent model of depression. Psychosom. Med. 70 (4), 435-443.

Grippo, A.J., Ihm, E., Wardwell, J., McNeal, N., Scotti, M.A., Moenk, D.A., Chandler, D.L., LaRocca, M.A., Preihs, K., 2014. The effects of environmental enrichment on depressive and anxiety-relevant behaviors in socially isolated prairie voles. Psychosom. Med. 76 (4), 277-284.

Grissom, N., Bhatnagar, S., 2009. Habituation to repeated stress: get used to it. Neurobiol. Learn. Mem. 92 (2), 215-224.

Habib, M., Shaker, S., El-Gayar, N., Aboul-Fotouh, S., 2015. The effects of antidepressants "fluoxetine and imipramine" on vascular abnormalities and Toll like receptor-4 expression in diabetic and non-diabetic rats exposed to chronic stress. PLoS One 10 (3), e0120559.

Haile, C.N., GrandPre, T., Kosten, T.A., 2001. Chronic unpredictable stress, but not chronic predictable stress, enhances the sensitivity to the behavioral effects of cocaine in rats. Psychopharmacology 154 (2), 213-220.

Handa, R.J., Burgess, L.H., Kerr, J.E., O'Keefe, J.A., 1994. Gonadal steroid hormone receptors and sex differences in the hypothalamo-pituitary-adrenal axis. Horm. Behav. 28 (4), 464-476.

Hay, M., 2016. Sex, the brain and hypertension: brain oestrogen receptors and high blood pressure risk factors. Clin. Sci. (Lond.) 130 (1), 9-18.

Helmreich, D.L., Morano, M.I., Akil, H., Watson, S.J., 1997. Correlation between changes in stress-induced corticosterone secretion and GR mRNA levels. Stress 2 (2), 101-112.

Herman, J.P., 2013. Neural control of chronic stress adaptation. Front. Behav. Neurosci. 7,61 .

Huynh, T.N., Krigbaum, A.M., Hanna, J.J., Conrad, C.D., 2011. Sex differences and phase of light cycle modify chronic stress effects on anxiety and depressive-like behavior. Behav. Brain Res. 222 (1), 212-222.

Isgor, C., Kabbaj, M., Akil, H., Watson, S.J., 2004. Delayed effects of chronic variable stress during peripubertal-juvenile period on hippocampal morphology and on cognitive and stress axis functions in rats. Hippocampus 14 (5), 636-648.

Isingrini, E., Belzung, C., Freslon, J.L., Machet, M.C., Camus, V., 2012. Fluoxetine effect on aortic nitric oxide-dependent vasorelaxation in the unpredictable chronic mild stress model of depression in mice. Psychosom. Med. 74 (1), 63-72.

Iwasaki-Sekino, A., Mano-Otagiri, A., Ohata, H., Yamauchi, N., Shibasaki, T., 2009. Gender differences in corticotropin and corticosterone secretion and corticotropinreleasing factor mRNA expression in the paraventricular nucleus of the hypothalamus and the central nucleus of the amygdala in response to footshock stress or psychological stress in rats. Psychoneuroendocrinology 34 (2), 226-237.

Junior, U.L., Cordellini, S., 2007. Differential vascular adaptive response to stress exposure in male and female rats: role of gonadal hormones and endothelial cells. Stress 10 (1), 27-36.

Kearney, P.M., Whelton, M., Reynolds, K., Muntner, P., Whelton, P.K., He, J., 2005 Global burden of hypertension: analysis of worldwide data. Lancet 365 (9455), 217-223.

Kessler, R.C., McGonagle, K.A., Zhao, S., Nelson, C.B., Hughes, M., Eshleman, S., Wittchen, H.U., Kendler, K.S., 1994. Lifetime and 12-month prevalence of DSM-III-R psychiatric disorders in the United States. Results from the National Comorbidity Survey. Arch. Gen. Psychiatry 51 (1), 8-19.

Koolhaas, J.M., Bartolomucci, A., Buwalda, B., de Boer, S.F., Flugge, G., Korte, S.M., Meerlo, P., Murison, R., Olivier, B., Palanza, P., Richter-Levin, G., Sgoifo, A., Steimer, T., Stiedl, O., van Dijk, G., Wohr, M., Fuchs, E., 2011. Stress revisited: a critical evaluation of the stress concept. Neurosci. Biobehav. Rev. 35 (5), 1291-1301.

Kudielka, B.M., Kirschbaum, C., 2005. Sex differences in HPA axis responses to stress: a review. Biol. Psychol. 69 (1), 113-132.

Laurent, S., Juillerat, L., London, G., Nussberger, J., Brunner, H., Safar, M., 1987. Hyperreactivity of the humeral artery to noradrenaline in essential hypertension patients. Arch. Mal. Coeur Vaiss. 80 (6), 826-831.

Ledvenyiova-Farkasova, V., Bernatova, I., Balis, P., Puzserova, A., Bartekova, M. Gablovsky, I., Ravingerova, T., 2015. Effect of crowding stress on tolerance to ischemia-reperfusion injury in young male and female hypertensive rats: molecular mechanisms. Can. J. Physiol. Pharmacol. 93 (9), 793-802.

Lenglos, C., Mitra, A., Guevremont, G., Timofeeva, E., 2013. Sex differences in the effects of chronic stress and food restriction on body weight gain and brain expression of 
CRF and relaxin-3 in rats. Genes Brain Behav. 12 (4), 370-387.

Lopez, M., Tena-Sempere, M., 2015. Estrogens and the control of energy homeostasis: a brain perspective. Trends Endocrinol. Metab. 26 (8), 411-421.

Lu, J., Wu, X.Y., Zhu, Q.B., Li, J., Shi, L.G., Wu, J.L., Zhang, Q.J., Huang, M.L., Bao, A.M., 2015. Sex differences in the stress response in SD rats. Behav. Brain Res. 284, 231-237.

Maeng, L.Y., Milad, M.R., 2015. Sex differences in anxiety disorders: interactions between fear, stress, and gonadal hormones. Horm. Behav. 76, 106-117.

Magarinos, A.M., McEwen, B.S., 1995. Stress-induced atrophy of apical dendrites of hippocampal CA3c neurons: comparison of stressors. Neuroscience 69 (1), 83-88.

Marin, M.T., Cruz, F.C., Planeta, C.S., 2007. Chronic restraint or variable stresses differently affect the behavior, corticosterone secretion and body weight in rats. Physiol. Behav. 90 (1), 29-35.

McCarty, R., 2016. Learning about stress: neural, endocrine and behavioral adaptations. Stress $1-30$.

Mitsushima, D., Masuda, J., Kimura, F., 2003. Sex differences in the stress-induced release of acetylcholine in the hippocampus and corticosterone from the adrenal cortex in rats. Neuroendocrinology 78 (4), 234-240.

Noschang, C.G., Pettenuzzo, L.F., von Pozzer Toigo, E., Andreazza, A.C., Krolow, R., Fachin, A., Avila, M.C., Arcego, D., Crema, L.M., Diehl, L.A., Goncalvez, C.A., Vendite, D., Dalmaz, C., 2009. Sex-specific differences on caffeine consumption and chronic stress-induced anxiety-like behavior and DNA breaks in the hippocampus. Pharmacol. Biochem. Behav. 94 (1), 63-69.

Padovan, C.M., Guimaraes, F.S., 2000. Restraint-induced hypoactivity in an elevated plusmaze. Braz. J. Med. Biol. Res. 33 (1), 79-83.

Pastor-Ciurana, J., Rabasa, C., Ortega-Sanchez, J.A., Sanchis-Olle, M., Gabriel-Salazar, M., Ginesta, M., Belda, X., Daviu, N., Nadal, R., Armario, A., 2014. Prior exposure to repeated immobilization or chronic unpredictable stress protects from some negative sequels of an acute immobilization. Behav. Brain Res. 265, 155-162.

Pellow, S., File, S.E., 1986. Anxiolytic and anxiogenic drug effects on exploratory activity in an elevated plus-maze: a novel test of anxiety in the rat. Pharmacol. Biochem. Behav. 24 (3), 525-529.

Perrot-Sinal, T.S., Gregus, A., Boudreau, D., Kalynchuk, L.E., 2004. Sex and repeated restraint stress interact to affect cat odor-induced defensive behavior in adult rats. Brain Res. 1027 (1-2), 161-172.

Porter, J.P., Phillips, A., Rich, J., Wright, D., 2004. Effect of chronic stress on the cardiac baroreflex in the post-weanling rat. Life Sci. 75 (13), 1595-1607.

Prut, L., Belzung, C., 2003. The open field as a paradigm to measure the effects of drugs on anxiety-like behaviors: a review. Eur. J. Pharmacol. 463 (1-3), 3-33.

Radley, J.J., Sawchenko, P.E., 2015. Evidence for involvement of a limbic paraventricular hypothalamic inhibitory network in hypothalamic-pituitary-adrenal axis adaptations to repeated stress. J. Comp. Neurol. 523 (18), 2769-2787.

Rivier, C., 1999. Gender, sex steroids, corticotropin-releasing factor, nitric oxide, and the HPA response to stress. Pharmacol. Biochem. Behav. 64 (4), 739-751. dos Santos, R.L., da Silva, F.B., Ribeiro Jr., R.F., Stefanon, I, 2014. Sex hormones in the cardiovascular system. Horm. Mol. Biol. Clin. Invest. 18 (2), 89-103.

Sarnyai, Z., Biro, E., Penke, B., Telegdy, G., 1992. The cocaine-induced elevation of plasma corticosterone is mediated by endogenous corticotropin-releasing factor (CRF) in rats. Brain Res. 589 (1), 154-156.

Solomon, M.B., Herman, J.P., 2009. Sex differences in psychopathology: of gonads, adrenals and mental illness. Physiol. Behav. 97 (2), 250-258.

Stanley, S.C., Brooks, S.D., Butcher, J.T., d'Audiffret, A.C., Frisbee, S.J., Frisbee, J.C., 2014. Protective effect of sex on chronic stress- and depressive behavior-induced vascular dysfunction in BALB/cJ mice. J. Appl. Physiol. (1985) 117 (9), 959-970.

Steptoe, A., Kivimaki, M., 2012. Stress and cardiovascular disease. Nat. Rev. Cardiol. 9 (6), 360-370.

Tang, E.H., Vanhoutte, P.M., 2010. Endothelial dysfunction: a strategic target in the treatment of hypertension? Pflugers Arch. 459 (6), 995-1004.

Ter Horst, G.J., Wichmann, R., Gerrits, M., Westenbroek, C., Lin, Y., 2009. Sex differences in stress responses: focus on ovarian hormones. Physiol. Behav. 97 (2), 239-249.

Toth, Z.E., Zelena, D., Mergl, Z., Kirilly, E., Varnai, P., Mezey, E., Makara, G.B., Palkovits, M., 2008. Chronic repeated restraint stress increases prolactin-releasing peptide/ tyrosine-hydroxylase ratio with gender-related differences in the rat brain. J. Neurochem. 104 (3), 653-666.

Vyas, A., Mitra, R., Shankaranarayana Rao, B.S., Chattarji, S., 2002. Chronic stress induces contrasting patterns of dendritic remodeling in hippocampal and amygdaloid neurons. J. Neurosci. 22 (15), 6810-6818.

Walf, A.A., Frye, C.A., 2005. Antianxiety and antidepressive behavior produced by physiological estradiol regimen may be modulated by hypothalamic-pituitary-adrenal axis activity. Neuropsychopharmacology 30 (7), 1288-1301.

Weinstock, M., Razin, M., Schorer-Apelbaum, D., Men, D., McCarty, R., 1998. Gender differences in sympathoadrenal activity in rats at rest and in response to footshock stress. Int. J. Dev. Neurosci. 16 (3-4), 289-295.

Weissman, M.M., Bland, R.C., Canino, G.J., Faravelli, C., Greenwald, S., Hwu, H.G., Joyce, P.R., Karam, E.G., Lee, C.K., Lellouch, J., Lepine, J.P., Newman, S.C., RubioStipec, M., Wells, J.E., Wickramaratne, P.J., Wittchen, H., Yeh, E.K., 1996. Crossnational epidemiology of major depression and bipolar disorder. JAMA 276 (4) 293-299.

Xing, Y., He, J., Hou, J., Lin, F., Tian, J., Kurihara, H., 2013. Gender differences in CMS and the effects of antidepressant venlafaxine in rats. Neurochem. Int. 63 (6), 570-575.

Xu, L., Bloem, B., Gaszner, B., Roubos, E.W., Kozicz, T., 2010. Stress-related changes in the activity of cocaine- and amphetamine-regulated transcript and nesfatin neurons in the midbrain non-preganglionic Edinger-Westphal nucleus in the rat. Neuroscience 170 (2), 478-488.

Yang, H.J., Kim, K.Y., Kang, P., Lee, H.S., Seol, G.H., 2014. Effects of salvia sclarea on chronic immobilization stress induced endothelial dysfunction in rats. BMC Complement. Altern. Med. 14, 396. 